\title{
SMALE FLOWS ON THE THREE-SPHERE
}

\author{
KETTY DE REZENDE
}

\begin{abstract}
In this paper, a complete classification of Smale flows on $S^{3}$ is obtained. This classification is presented by means of establishing a concise set of properties that must be satisfied by an (abstract) Lyapunov graph associated to a Smale flow and a Lyapunov function. We show that these properties are necessary, that is, given a Smale flow and a Lyapunov function, its Lyapunov graph satisfies this set of properties. We also show that these properties are sufficient, that is, given an abstract Lyapunov graph $L^{\prime}$ satisfying this set of properties, it is possible to realize a Smale flow on $S^{3}$ that has a graph $L$ as its Lyapunov graph where $L$ is equal to $L^{\prime}$ up to topological equivalence. The techniques employed in proving that the conditions imposed on the graph are necessary involve some use of homology theory. Geometrical methods are used to construct the flow on $S^{3}$ associated to the given graph and therefore establish the sufficiency of the above conditions. The main theorem in this paper generalizes a result of Franks [8] who classified nonsingular Smale flows on $S^{3}$.
\end{abstract}

1. Introduction. The qualitative study of a class of dynamical systems consists in firstdescribing geometrically the behavior of the flow on the basic sets where ultimately the dynamics of the system is concentrated. Secondly, in describing the disposition of the basic sets on the manifold.

In this context, the Lyapunov graph becomes a useful tool since it gives a global picture of how the basic sets are situated on the manifold.

DEFINITION 1.1. A Lyapunov graph $L$ for a flow $\phi_{t}: M \rightarrow M$ and a Lyapunov function $f: M \rightarrow \mathbf{R}$ is obtained by taking the quotient complex of $M$ by identifying to a point each component of a level set of $f$.

Each vertex corresponds to a critical level of $f$, i.e., to a basic set of $\phi_{t}$. Each edge corresponds to a component of the inverse image of $f$ of an open interval. This component is diffeomorphic to $N \times(0,1)$ where $N$ is a codimension one submanifold of $M$. Each edge is labelled with the genus of $N$.

In order to construct examples of flows that satisfy certain dynamical data, it is useful to record such data on an abstract Lyapunov graph.

DEFINITION 1.2. An abstract Lyapunov graph is a finite connected oriented graph $L$ which possesses no oriented cycles and with each vertex labelled with a chain recurrent flow on a compact space. Each edge will be labelled with a nonnegative integer $g$, which we refer to as the weight on the edge.

The dominating theme throughout this paper is the realization of an abstract Lyapunov graph. In other words, given an abstract Lyapunov graph and a manifold

Received by the editors November 18, 1985 and, in revised form, September 19, 1986.

1980 Mathematics Subject Classification (1985 Revision). Primary 58F15; Secondary 58F99.

Research supported in part by CNPq-Conselho Nacional de Desenvolvimento Científico e Tecnológico (Brasil). 
$M$ we wish to construct a flow on $M$ that has a Lyapunov graph $L^{\prime}$ topologically equivalent to $L$. Alongside the realization problem it is equally important and interesting to determine properties that a Lyapunov graph associated to a flow and a Lyapunov function satisfy.

Our approach to the realization problem is to construct a flow on a submanifold of $M$ which possesses only one basic set. This flow will realize the part of $L$ consisting of a vertex $v$ and its $e^{+}$entering edges and $e^{-}$exiting edges. This submanifold will have $e^{+}$boundary components where the flow enters, $e^{-}$boundary components where the flow exits and will satisfy all additional requirements that the graph imposes. The last step will consist of gluing these submanifolds to obtain $M$ and a smooth flow on $M$.

In this paper we are dealing with Smale flows which by definition have all basic sets of dimension $\leq 1$. Basic sets of dimension equal to one are fully understood in terms of shift maps by the following classification theorem due to Bowen [3]:

THEOREM 1.3. If $\phi_{t}$ is a flow with hyperbolic chain recurrent set and $\Lambda$ is a one-dimensional basic set, then $\phi_{t}$ restricted to $\Lambda$ is topologically equivalent to the suspension of a basic subshift of finite type (i.e., a subshift associated to an irreducible matrix).

This theorem enables us to label the vertices of the (abstract) Lyapunov graph that represent the one-dimensional basic sets of a Smale flow with the suspension of a subshift of finite type $\sigma(A)$. For simplicity we will label the vertex with the nonnegative integer irreducible matrix $A$.

The question of the realization of the suspension of a basic subshift of finite type as a one-dimensional basic set on a given manifold was settled by Williams [13] for manifolds of dimension $\geq 4$ :

THEOREM 1.4. The suspension of any basic subshift of finite type is topologically equivalent to a basic set of some Smale flow on any $m$-manifold, $m \geq 4$.

A sharper result was later obtained by Pugh and Shub [9] for $m \geq 3$.

Our problem is of a more global nature since it involves the realization of a Lyapunov graph which is labelled with a collection of suspensions of subshifts of finite type together with a collection of singularities as a Smale flow on an $m$ manifold.

I would like to thank several people for helpful conversations, in particular my thesis adviser John Franks who suggested the problem and the referee who read the paper in great detail and made several good suggestions.

2. Preliminaries. For a more detailed account of the following material we refer the reader to the book written by J. Franks, Homology and Dynamical Systems [7].

Recall that for a flow $\phi_{t}$ on $M$, a point $x \in M$ is a chain recurrent point if for any $\varepsilon>0$ there exists an $\varepsilon$-chain from $x$ to $x$, that is, if there exists $x_{1}=x, x_{2}, \ldots$, $x_{n}=x$ and real numbers $t(i)>0$ such that $d\left(\phi_{t(i)}\left(x_{i}\right), x_{i+1}\right)<\varepsilon$ for $1 \leq i<n$. The set of chain recurrent points will be called the chain recurrent set and will be denoted by $R$ or $R\left(\phi_{t}\right)$.

The importance of considering the chain recurrent set for a continuous flow $\phi_{t}$ is reflected by a theorem of Conley [6] which guarantees the existence of a function that decreases along the orbit of $x \notin R\left(\phi_{t}\right)$ and is constant for $x, y \in R\left(\phi_{t}\right)$ provided there is an $\varepsilon$-chain from $x$ to $y$. 
THEOREM 2.1. If $\phi_{t}$ is a continuous flow on $M$ then there exists a continuous function $g: M \rightarrow \mathbf{R}$ such that:

(1) If $x \notin R\left(\phi_{t}\right)$ then $g\left(\phi_{t}(x)\right)<g\left(\phi_{s}(x)\right)$ for $t>s$.

(2) If $x, y \in R\left(\phi_{t}\right)$ then $g(x)=g(y)$ if and only if for $\varepsilon>0, \exists$ points $x_{1}=x, x_{2}, \ldots, x_{n}=y, x_{n+1}, \ldots, x_{2 n}=x$ in $R\left(\phi_{t}\right)$ and real numbers $t(i)>0$ such that $d\left(\phi_{t(i)}\left(x_{i}\right), x_{i+1}\right)<\varepsilon$ for $1 \leq i<2 n$.

A Lyapunov function is a function $g: M \rightarrow \mathbf{R}$ that satisfies (1) and (2) above.

Before we define Smale flow we need the following definitions.

DEFINITION 2.2. The chain recurrent set $R$ has hyperbolic structure provided that the tangent bundle restricted to $R, T_{R} M$ is the Whitney sum of three bundles $E^{u} \oplus E^{s} \oplus E^{c}$, where $E^{c}$ is spanned by the vector field generating $\phi_{t}$ and $\exists$ constants $C>1$ and $a>0$ such that:

$$
\begin{gathered}
\left\|D \phi_{t}(v)\right\| \leq C e^{-a t}\|v\| \quad \text { for } v \in E^{s} \text { and } t \geq 0 \\
\left\|D \phi_{t}(v)\right\| \geq C^{-1} e^{a t}\|v\| \quad \text { for } v \in E^{u} \text { and } t \geq 0 .
\end{gathered}
$$

An important consequence of the hyperbolic structure of the chain recurrent set $R$ is Smale's Spectral Decomposition Theorem [12]:

THEOREM 2.3. Suppose that the chain recurrent set $R$ of a flow on a compact manifold $\phi_{t}: M \rightarrow M$ has a hyperbolic structure. Then $R$ is a finite disjoint union of basic sets $\Lambda_{1}, \Lambda_{2}, \ldots, \Lambda_{n}$.

A basic set $\Lambda$ is a compact invariant set that contains a point whose forward orbit is dense in $\Lambda$. The index of a basic set $\Lambda$ is the fiber dimension of the bundle $\left.E^{u}\right|_{\Lambda}$

The existence of a smooth Lyapunov function for a flow $\phi_{t}$ implies the existence of a filtration associated to it. If $\phi_{t}: M \rightarrow M$ is a flow with hyperbolic chain recurrent set and $\left\{\Lambda_{i}\right\}_{i=1}^{n}$ are its basic sets then a filtration associated to $\phi_{t}$ is a collection of compact submanifolds $M_{0} \subset M_{1} \subset \cdots \subset M_{n}=M$ such that $\phi_{t}\left(M_{i}\right) \subset$ int $M_{i}$, for all $t>0$ and $\Lambda_{i}=\bigcap_{t=-\infty}^{\infty} \phi_{t}\left(M_{i}-M_{i-1}\right)$.

An important notion is that of the transversality of the unstable manifold at $x$ with the stable manifold at $y$ for all $x, y$ in the hyperbolic chain recurrent set $R$. This is known as the transversality condition and is a necessary condition for structural stability.

DEFINITION 2.4. A smooth flow $\phi_{t}: M \rightarrow M$ on a compact manifold is called a Smale flow if:

(1) the chain recurrent set $R$ has hyperbolic structure;

(2) the $\operatorname{dim} R \leq 1$;

(3) $\phi_{t}$ satisfies the transversality condition.

Symbolic Dynamics has played a major role in the study of structurally stable dynamical systems. Let us recall some definitions that we will use frequently throughout this paper.

Let $A^{n \times n}$ be a nonnegative integer matrix. Form a graph $G$ with $n$ vertices and $a_{i j}$ oriented edges going from vertex $i$ to vertex $j$. Let $E=\left\{e_{i}\right\}$ be the set of edges. Let $E^{Z}$ be the space of sequences of elements of $E$ indexed by Z. Define $\Sigma_{A} \subset E^{Z}$ by: $\underline{e}=\left(\ldots e_{-1}, e_{0}, e_{1} \ldots\right) \in \Sigma_{A}$ provided that for each $i \in \mathbf{Z}$ the oriented 
edge $e_{i}$ ends at the vertex of $G$ where $e_{i+1}$ begins. Define a shift homeomorphism $\sigma: E^{Z} \rightarrow E^{Z}$ by $\sigma(\underline{e})=\underline{f}$ where $f_{i}=e_{i-1}$.

DEFINITION 2.5. A subshift of finite type associated to a matrix $A^{n \times n}$ is the shift homeomorphism defined above restricted to $\Sigma_{A}, \sigma \mid \Sigma=\sigma(A): \Sigma_{A} \rightarrow \Sigma_{A}$.

DEFINITION 2.6. The suspension flow of the subshift of finite type (the suspension of the subshift of finite type) is defined on the space $X_{A}=\Sigma_{A} \times \mathbf{R} /[(x, s+1) \sim$ $(\sigma(x), s)]$ and the flow $\phi_{t}$ on $X_{A}$ is defined by $\phi_{t}(x, s)=(x, s+t)$.

If $A$ and $B$ are nonnegative integer matrices they are flow equivalent provided the suspension flows of the subshifts of finite type $\sigma(A)$ and $\sigma(B)$ are topologically equivalent.

We will be interested in matrices that are associated to subshifts of finite type and by Theorem 1.3, irreducibility is a necessary condition. A nonnegative $n \times n$ integer matrix is called irreducible provided that for each $1 \leq i, j \leq n$ there is a $k>0$ with $\left(A^{k}\right)_{i j} \neq 0$.

Let us recall a few elementary definitions from Graph Theory. For a more detailed account on the subject the reader is referred to [2].

A graph $G(V, E)$ is an ordered pair of disjoint sets $(V, E)$ such that $E \subset V \times V$. The elements of $V$ are called vertices and those of $E$ are called edges. Given an edge $e=\{u, v\} \in E$, we say that $u$ and $v$ are adjacent and that $e$ is incident on $u$ and $v$. The degree of a vertex $u \in V$ is the number of edges incident on $u$. A tree is a cycle free connected graph.

A graph $G(V, E)$ is said to be oriented if its edges are ordered pairs. An ordered pair $(u, v) \in E$ is called a directed edge from $u$ to $v$. We also say that the edge $e=(u, v)$ is an incoming edge of $v$ and is an outgoing edge of $u$. The indegree of a vertex $u$ is the number of incoming edges of $u$ and the outdegree of $u$ is the number of outgoing edges of $u$. Given an oriented graph $G(V, E)$ we refer to the underlying graph of $G$ as being the graph $G(V, E)$ where the elements of the edge set $E$ are regarded as unordered pairs of vertices.

Two graphs $G(V, E)$ and $G^{\prime}\left(V^{\prime}, E^{\prime}\right)$ are said to be isomorphic if $\exists$ a bijection $\phi: V \rightarrow V$ that preserves adjacency relations, that is, $\forall v, w \in V,\{\phi(v), \phi(w)\} \in$ $E^{\prime} \Leftrightarrow\{v, w\} \in E$.

We will now define topological equivalence between two graphs.

DEFINITION 2.7. Let $G(V, E)$ and $G^{\prime}\left(V^{\prime}, E^{\prime}\right)$ be abstract Lyapunov graphs with nonnegative integer weight functions $w$ and $w^{\prime}$, respectively, associated to their edges, i.e., $w: E \rightarrow \mathbf{Z}^{+}$and $w^{\prime}: E^{\prime} \rightarrow \mathbf{Z}^{+}$. We say that $G$ and $G^{\prime}$ are topologically equivalent if the following hold:

(1) There is a weight preserving isomorphism $\phi$ between $G(V, E)$ and $G^{\prime}\left(V^{\prime}, E^{\prime}\right)$; that is, $w(u, v)=w^{\prime}(\phi(u), \phi(v))$.

(2) The chain recurrent flow that $v$ is labelled with is topologically equivalent to the chain recurrent flow that $\phi(v)$ is labelled with.

In proving that the conditions of Theorem 3.1 are sufficient we will be involved in gluing a collection of handlebodies in order to build up $S^{3}$. A handlebody is any space obtained from the 3 -ball $D^{3}$ by attaching $g$ distinct copies of $D^{2} \times[-1,1]$ with homeomorphisms throwing the $2 g$ disks $D^{2} \times \pm 1$ onto $2 g$ disjoint 2-disks on $\partial D^{3}$ (all to be done in such a way that the resulting 3 -manifold is orientable). The integer $g$ is called the genus. A subset $X \subset Y$ is said to be collared (in $Y$ ) if there 


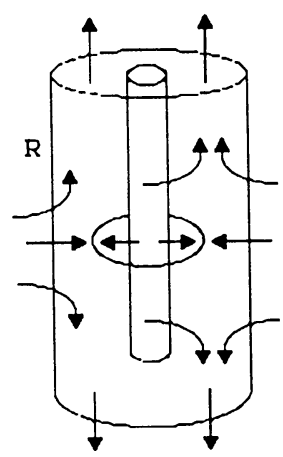

FIGURE 2.1

exists an embedding $b: X \times[0,1] \rightarrow Y$ such that $b(x, 0)=x \forall x \in X$. The image of $b$ is the collar $[\mathbf{1 0}]$.

Given a handlebody $M$, we will define a collared handlebody $\mathbf{M}$ to be $M$ union a collaring of $\partial M$ (this should not be confused with a collaring of the handlebody $M)$.

We will also need to define the concept of round handles [1].

DEFINITION 2.8. Let $C_{1}$ and $C_{2}$ be solid concentric cylinders with $C_{2} \subset C_{1}$. $A$ round handle $R$ is a 3-manifold homeomorphic to $C_{1}-C_{2}$ containing a saddle type periodic orbit of period equal to one and with a flow defined on $R$ as follows: the flow enters on two disjoint boundary components of $R$ homeomorphic to annuli and exits on two other disjoint boundary components also homeomorphic to annuli. See Figure 2.1.

We will now describe some ways in which to glue a round handle $R$ to collared handlebodies. Let $A$ and $B$ be the two annuli of $R$ where the flow exits and consider handlebodies $M_{1}$ and $M_{2}$ and the respective collared handlebodies $\mathbf{M}_{1}$ and $\mathbf{M}_{2}$. Let $p$ be the genus of $M_{1}$ and $q$ be the genus of $M_{2}$. The collar $\mathbf{M}_{i}-M_{i}$ is diffeomorphic to $\partial M \times[0, \delta)$. On this collar, the vector field tangent to the flow corresponds to $\frac{\partial}{\partial t}$, where $t$ is the coordinate on $[0, \delta)$. The flow enters through $\partial \mathbf{M}_{i}$ and exits on $\partial M_{i}, \quad i=1,2$. Let $X=\left(\mathbf{M}_{2}-M_{2}\right) \cup R \cup\left(\mathbf{M}_{1}-M_{1}\right)$.

The round handle $R$ will be called a connecting invariant handle when the annuli $A$ and $B$ are glued onto annuli $A^{\prime}$ and $B^{\prime}$ on $\partial \mathbf{M}_{1}$ and $\partial \mathbf{M}_{2}$ respectively. Furthermore, $A^{\prime}$ must contain a separating curve of $\partial \mathbf{M}_{1}$ and similarly $B^{\prime}$. Notice that $\partial\left(\mathbf{M}_{1} \cup R \cup \mathbf{M}_{2}\right)$ has two components that correspond to the entering boundary of $X$. Both components will be 2-manifolds of genus $r$ and $s$ such that $r+s=p+q$. Notice that the sum of the genera of the components of the exiting boundary of $X$ remains the same for the components of the entering boundary of $X$. (See Figure 2.2.)

The round handle $R$ will be called a connecting decreasing handle when annulus $A$ is glued onto an annulus $A^{\prime}$ on $\partial \mathbf{M}_{1}$ where $A^{\prime}$ must contain a nonseparating curve of $\partial \mathbf{M}_{1}$. Also, annulus $B$ must be glued onto annulus $B^{\prime}$ on $\partial \mathbf{M}_{2}$ where $B^{\prime}$ must contain a homotopically trivial curve of $\partial \mathbf{M}_{2}$. Notice that $\partial\left(\mathbf{M}_{1} \cup R \cup \mathbf{M}_{2}\right)$ has one boundary component of genus $p+q-1$, which corresponds to the entering boundary of $X$. Therefore, the sum of the genera of the components of the exiting 


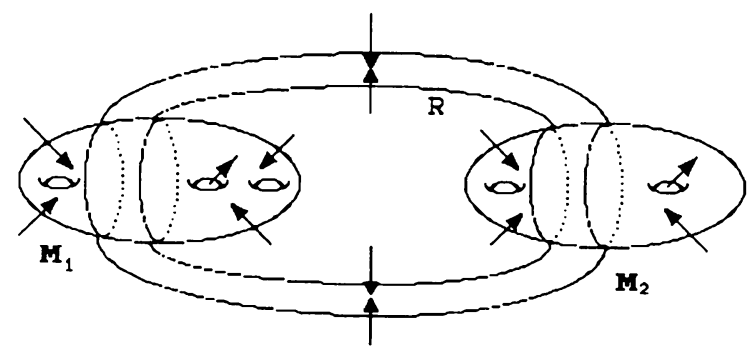

FIGURE 2.2

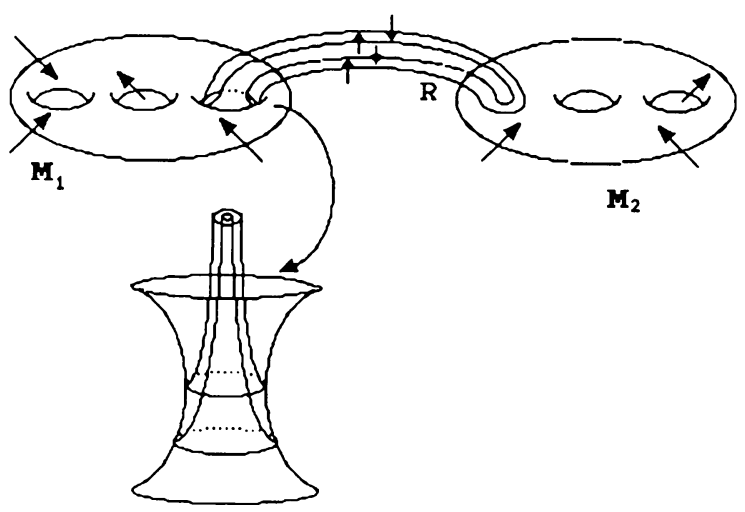

FIGURE 2.3

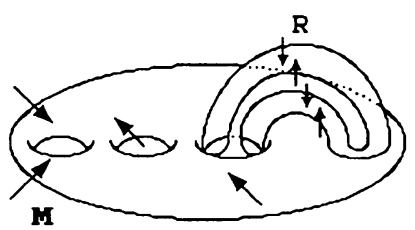

FIGURE 2.4

boundary is decreased by one for the genus of the entering boundary of $X$. (See Figure 2.3.)

It will also be of interest to consider gluing the round handle $R$ to a collared handlebody of genus $p$. Define a flow on $\mathbf{M}-M$ that enters on $\partial \mathbf{M}$ and exits on $\partial M$. Let $X=R \cup(\mathbf{M}-M)$.

The round handle $R$ will be called an invariant handle if the annulus $A$ is glued onto an annulus $A^{\prime}$ on $\partial \mathbf{M}$ which contains a nonseparating curve. Also, the annulus $B$ must be glued onto an annulus $B^{\prime} \subset \partial \mathbf{M}$ which is homotopically trivial. Note that the entering boundary of $X$, i.e., $\partial(\mathbf{M} \cup R)$, remains with the same genus as the exiting boundary of $X$, i.e., $\partial M$. (See Figure 2.4.)

A nilpotent handle is homeomorphic to $D^{1} \times D^{1} \times D^{1}$ and contains no chain recurrent point. This handle is embedded in the collar of a handlebody $M$ i.e., in $\mathbf{M}-M$. Define the flow on the nilpotent handle to be the same as the flow on the collars, so there is no problem in the resulting flow due to the embedding. 
3. Main result. We will characterize necessary and sufficient conditions that should be imposed on a Lyapunov graph so that it is associated to a Smale flow on $S^{3}$. This problem in such a general setting was first studied by Franks for nonsingular Smale flows on $S^{3}$. He characterized necessary and sufficient conditions on a Lyapunov graph associated to a nonsingular Smale flow on $S^{3}$. The following result is a generalization of a theorem obtained by Franks in [8].

THEOREM 3.1. Given an abstract Lyapunov graph L whose sink (source) vertices are each labelled with an attracting (repelling) periodic orbit or an index 0 (index 3) singularity, then $L$ is associated with a Smale flow $\phi_{t}$ and a Lyapunov function $f$ on $S^{3}$ if and only if the following conditions hold:

(1) The underlying graph $L$ is a tree with exactly one edge attached to each sink or source vertex.

(2) If a vertex is labelled with a singularity of index 2 (index 1) then $1 \leq e^{+} \leq 2$ and $e^{-}=1\left(e^{+}=1\right.$ and $\left.1 \leq e^{-} \leq 2\right)$.

If a vertex is labelled with a suspension of a subshift of finite type and $A^{m \times m}$ is the nonnegative integer matrix representing this subshift, then

$$
\begin{gathered}
e^{+}>0, \quad e^{-}>0, \\
k+1-G^{-} \leq e^{+} \leq k+1, \quad \text { with } G^{-}=\sum_{i=1}^{e^{-}} g_{i}^{-} \text {and } \\
k+1-G^{+} \leq e^{-} \leq k+1, \quad \text { with } G^{+}=\sum_{j=1}^{e^{+}} g_{j}^{+}
\end{gathered}
$$

where $k=\operatorname{dim} \operatorname{ker}\left((I-B): F_{2}^{m} \rightarrow F_{2}^{m}\right), F_{2}=\mathbf{Z} / 2$ and $b_{i j}=a_{i j} \bmod 2, e^{+}\left(e^{-}\right)$is the number of incoming (outgoing) edges of the vertex and $g_{j}^{+}\left(g_{i}^{-}\right)$is the weight on an incoming (outgoing) edge of the vertex.

(3) All vertices must satisfy the Poincaré-Hopf condition, i.e., for a vertex labelled with a singularity of index $r$, the condition is

$$
(-1)^{r}=e^{+}-e^{-}-\sum g_{j}^{+}+\sum g_{i}^{-}
$$

and for a vertex labelled with a suspension of a subshift of finite type or a periodic orbit, the condition is

$$
0=e^{+}-e^{-}-\sum g_{j}^{+}+\sum g_{i}^{-} .
$$

4. Proof of the necessity of the conditions. Given a Smale flow $\phi_{t}$ on $S^{3}$ with Lyapunov function $g: S^{3} \rightarrow \mathbf{R}$ we wish to show that the Lyapunov graph $L$ satisfies conditions 1,2 and 3 of Theorem 3.1.

The fact that $L$ is a tree follows from a result in [8]:

PROPOSITION 4.1. Let $M$ be a compact oriented manifold. Suppose that $\phi_{t}: M \rightarrow M$ is a smooth flow and $g: M \rightarrow \mathbf{R}$ is a Lyapunov function with an associated finite graph $L$. If $H_{1}(M ; \mathbf{Q})=0$ then $L$ is a tree.

We now proceed to prove the bounds on the indegree and outdegree of a given vertex. The methods employed here are also used in [8]. Homology theory plays 
an important role at this point. The main idea in this proof is the analysis of the dimensions of the homology groups of a pair $(X, Y)$ where $X$ and $Y$ are obtained from a filtration associated to $\phi_{t}$. (Note that $X$ and $Y$ are not necessarily part of a filtration associated to $\phi_{t}$.)

Let $\Lambda$ be a basic set of $\phi_{t}$ and $c=g(\Lambda)$ where $g$ is the Lyapunov function. Choose $\varepsilon>0$ sufficiently small so that $c$ is the only critical value in $[c-\varepsilon, c+\varepsilon]$. Let $X_{0}=g^{-1}(-\infty, c+\varepsilon], Y_{0}=g^{-1}(-\infty, c-\varepsilon]$ and $Z$ be the component of $X_{0}-Y_{0}$ which contains $\Lambda$. Now, let $Y$ be the union of the components of $S^{3}-\left(\partial Z \cap Y_{0}\right)$ which do not contain $\Lambda$ and $X=Y \cup Z$. Hence $X-Y$ is a neighborhood of $\Lambda$ whose boundary consists of surfaces. The flow exits on $e^{-}$surfaces and enters on $e^{+}$surfaces each of genus $g_{i}^{-}$and $g_{j}^{+}$respectively. Thus, $Y$ has $e^{-}$components $Y_{i}$ each bounded by a surface of genus $g_{i}^{-}$. Consequently, each $Y_{i}$ has the homology of a handlebody.

Consider the exact homology sequence for the pair $(X, Y)$ :

$$
\begin{aligned}
H_{2}(Y) & \rightarrow H_{2}(X) \rightarrow_{\beta} H_{2}(X, Y) \rightarrow \delta H_{1}(Y) \\
& \rightarrow \eta H_{1}(X) \rightarrow H_{1}(X, Y) \rightarrow H_{0}(Y) \rightarrow_{\alpha} H_{0}(X) \rightarrow 0 .
\end{aligned}
$$

In this general setting, we will proceed to compute the dimensions of some of the homology groups above for different basic sets. We remark that we will be using field coefficients F. For this we will invoke the Alexander Duality Theorem:

THEOREM 4.2. Let $A$ be a compact submanifold of $S^{n}$. Then

$$
H^{q}(A) \simeq \tilde{H}_{n-q-1}\left(S^{n}-A\right) \text { for all } q<n
$$

(where $\tilde{H}_{*}$ denotes reduced homology).

Let us begin by considering the case where $\Lambda$ is taken to be a singularity. We remark that locally around a singularity $p$ we may assume that $\phi_{t}$ is gradient-like with respect to the Lyapunov function $g$ (see [11]). As a corollary of Proposition 2.6 in [7] we obtain the following lemma:

LEMMA 4.3. The manifold $X_{0}$ has $Y_{0} \cup W_{\varepsilon}^{u}(p)$ as a strong deformation retract.

The manifolds we are considering are $X=X_{0} \cup W$ and $Y=Y_{0} \cup W$ where $W=Y_{0}^{c}-(Z \cup C)$ and $C$ is the union of the submanifolds which intersect $\left(\overline{X_{0}^{c}} \cap \partial \mathbf{Z}\right)$. $Y_{0}^{c}$ denotes the complement of $Y_{0}$.

PROPOSITION 4.4. If $p$ is a singularity of index $k$ of the Smale flow $\phi_{t}$ and $X$ and $Y$ are defined as above, then

$$
H_{i}(X, Y ; \mathbf{F})= \begin{cases}\mathbf{F} & \text { if } i=k \\ 0 & \text { if } i \neq k\end{cases}
$$

where $\mathbf{F}$ is a field.

ProOF. Since $X$ has $Y \cup W_{\varepsilon}^{u}(p)$ as a strong deformation retract,

$$
H_{i}(X, Y ; \mathbf{F}) \simeq H_{i}\left(Y \cup W_{\varepsilon}^{u}(p), Y ; \mathbf{F}\right) \text {. }
$$

By excision,

$$
H_{i}\left(Y \cup W_{\varepsilon}^{u}(p), Y ; \mathbf{F}\right) \simeq H_{i}\left(W_{\varepsilon}^{u}(p), \partial W_{\varepsilon}^{u}(p) ; \mathbf{F}\right) \simeq\left\{\begin{array}{ll}
\mathbf{F} & \text { if } i=k, \\
0 & \text { if } i \neq k .
\end{array} \quad\right. \text { Q.E.D. }
$$


If $\Lambda$ is a source, by Proposition 4.4 $H_{1}(X, Y)=0$. Therefore, the map $\alpha: H_{0}(Y)$ $\rightarrow H_{0}(X)$ is an isomorphism. Consequently $1=\operatorname{dim} H_{0}(X)=\operatorname{dim} H_{0}(Y)=e^{-}$. Similarly if $\Lambda$ is a sink, by reversing the flow one obtains that $e^{+}=1$.

Now let us consider $\Lambda$ to be a singularity of index 2 . Since each $Y_{i}$ has the homology of a handlebody, this implies that $H_{2}\left(Y_{i}\right)=0$. Consequently, $H_{2}(Y)=0$ since $Y=\bigcup Y_{i}$. By the Alexander Duality Theorem $H^{2}(X) \simeq \tilde{H}_{0}\left(S^{3}-X\right)$ which implies that the dimensions of the two groups are equal. Also, $\operatorname{dim} H^{2}(X)=$ $\operatorname{dim} \tilde{H}_{0}\left(S^{3}-X\right)=e^{+}-1$ since $S^{3}-X$ has $e^{+}$components. In view of the fact that we are using field coefficients, $\operatorname{dim} H^{2}(X)=\operatorname{dim} H_{2}(X)$. Note that by Proposition 4.4 we have that $\operatorname{dim} H_{2}(X, Y)=1$.

By the preceding remarks, namely that $H_{2}(Y)=0$ and by exactness, it follows that the map $\beta: H_{2}(X) \rightarrow H_{2}(X, Y)$ is injective and therefore $\operatorname{dim} H_{2}(X)=$ $e^{+}-1 \leq \operatorname{dim} H_{2}(X, Y)=1$.

Using Proposition 4.4 once more, we obtain that $\operatorname{dim} H_{1}(X, Y)=0$. This means that the map $\alpha: H_{0}(X) \rightarrow H_{0}(Y)$ is an isomorphism which implies that $e^{-}=$ $\operatorname{dim} H_{0}(Y)=\operatorname{dim} H_{0}(X)=1$. This establishes the desired bounds $1 \leq e^{+} \leq 2$ and $e^{-}=1$. We remark that by reversing the flow on $Z$ of an index 2 singularity one obtains the bounds for an index 1 singularity.

Let us now turn to the case where $\Lambda$ is a one-dimensional basic set which is either a saddle type periodic orbit or a suspension of a subshift of finite type. To this end we state the following result due to Bowen and Franks [4]:

THEOREM 4.5. If $\phi_{t}$ is a Smale flow with associated filtration $\left\{M_{j}\right\}$ and $B$ is an $n \times n$ structure matrix for the basic set $\Lambda_{i}$ of index $u$ then

$$
\begin{gathered}
H_{k}\left(M_{i}, M_{i-1} ; \mathbf{F}\right)=0 \quad \text { if } k \neq u, u+1, \\
H_{u}\left(M_{i}, M_{i-1} ; \mathbf{F}\right)=\mathbf{F}^{n} /(I-B) \mathbf{F}^{n}, \\
H_{u+1}\left(M_{i}, M_{i-1} ; \mathbf{F}\right)=\operatorname{ker}\left((I-B) \text { on } \mathbf{F}^{n}\right)
\end{gathered}
$$

for any abelian group $\mathbf{F}$.

Lemma 4.6. Let $X_{0}, Y_{0}, X$ and $Y$ be as defined above. Then $H_{2}\left(X_{0}, Y_{0}\right) \simeq$ $H_{2}(X, Y)$. that

ProOF. By excising $U=X \cap X_{0}^{c}$ where $X_{0}^{c}$ is the complement of $X_{0}$, we have

$$
H_{2}(X, Y) \simeq H_{2}(X-U, Y-U)=H_{2}\left(X_{0}, Y-U\right) .
$$

Since $Y_{0}$ is a strong deformation retract of $Y-U$ this implies that

$$
H_{2}(X, Y) \simeq H_{2}\left(X_{0}, Y_{0}\right) \text {. Q.E.D. }
$$

By Lemma 4.6, $\operatorname{dim} H_{2}(X, Y)=\operatorname{dim} H_{2}\left(X_{0}, Y_{0}\right)$. Using Theorem 4.5 we obtain that $\operatorname{dim} H_{2}(X, Y)=k$. The dimensions of $H_{2}(X)$ and of $H_{2}(Y)$ which are $e^{-}-1$ and 0 respectively, are obtained by the exact same arguments provided above. In view of these computations and by exactness of the long exact sequence we conclude that the map $\beta: H_{2}(X) \rightarrow H_{2}(X, Y)$ is injective. This implies the following inequality: $e^{+}-1=\operatorname{dim} H_{2}(X) \leq H_{2}(X, Y)=k$. Therefore $e^{+} \leq k+1$. We will now prove that $e^{+} \geq k+1-G^{-}$by showing that $0 \leq \operatorname{dim} \operatorname{Im} \eta=G^{-}-k+e^{+}-1$. Note that since $\operatorname{dim} \operatorname{ker} \beta=0$,

$$
\operatorname{dim} \operatorname{Im} \beta=\operatorname{dim} H_{2}(X)-\operatorname{dim} \operatorname{ker} \beta=e^{+}-1 .
$$


By exactness $\operatorname{dim} \operatorname{ker} \delta=\operatorname{dim} \operatorname{Im} \beta$. Hence:

$$
\operatorname{dim} \operatorname{Im} \delta=\operatorname{dim} H_{2}(X, Y)-\operatorname{dim} \operatorname{ker} \delta=k-\left(e^{+}-1\right) .
$$

We also have by exactness that $\operatorname{dim} \operatorname{ker} \eta=\operatorname{dim} \operatorname{Im} \delta$. This finally implies that

$$
\operatorname{dim} \operatorname{Im} \eta=\operatorname{dim} H_{1}(Y)-\operatorname{dim} \operatorname{ker} \eta=G^{-}-\left(k-e^{+}+1\right) .
$$

The upper bound for $e^{-}$is obtained by reversing the flow which interchanges the roles of $e^{+}$and $e^{-}$. It also changes the matrix from $A$ to $A^{t}$ but this leaves $k$ invariant. The lower bound for $e^{-}$will follow from the Poincaré-Hopf formula which we will discuss subsequently. This formula asserts that: $G^{+}+e^{-}=G^{-}+e^{+}$. This implies that $G^{+}+e^{-}-k-1=G^{-}+e^{+}-k-1 \geq 0$ giving us the desired bound.

We will now prove the Poincaré-Hopf condition for a given vertex $v$. It will provide us with restrictions on the sum of the weights of the incoming and outgoing edges of the vertex $v$. We are able to proceed via the Poincaré-Hopf formula:

THEOREM 4.7. Suppose $\phi_{t}$ is a smooth flow on an odd-dimensional manifold $M$ exiting through $\partial M^{-}$and entering on $\partial M^{+}$, where $\partial M=\partial M^{+} \cup \partial M^{-}$. Then $\sum I_{p}=\frac{1}{2}\left(\chi\left(\partial M^{+}\right)-\chi\left(\partial M^{-}\right)\right)$where the summation is taken over all singularities in $M, I_{p}$ is the index of the singularity $p$ and $\chi$ denotes the Euler characteristic.

PROOF. The standard Poincaré-Hopf formula asserts that $\sum I_{p}=\chi\left(M, \partial M^{-}\right)$. By the exact sequence of the pair $\left(M, \partial M^{-}\right)$we have that $\chi\left(M, \partial M^{-}\right)=\chi(M)-$ $\chi\left(\partial M^{-}\right)$. Consider two copies of $M$ and glue them along their boundaries identifying corresponding boundary points. The manifold $D$ obtained in this form is called the double of $M$. Since $D$ is an odd-dimensional compact oriented manifold without boundary the $\chi(D)=0$. Also $\chi(D)=2 \chi(M)-\chi(\partial M)$. Thus, $\chi(M)=\frac{1}{2} \chi(\partial M)$. Therefore, by the previous remarks

$$
\begin{aligned}
\sum I_{p} & =\chi(M)-\chi\left(\partial M^{-}\right)=\frac{1}{2} \chi(\partial M)-\chi\left(\partial M^{-}\right) \\
& =\frac{1}{2}\left(\chi\left(\partial M^{+}\right)-\chi\left(\partial M^{-}\right)\right) . \quad \text { Q.E.D. }
\end{aligned}
$$

Let us choose $Z$ as defined previously to be the 3-manifold in the theorem above. Recall, that by definition, $Z$ has only one basic set.

If the basic set in $Z$ is a source, by the formula we obtain

$$
(-1)^{3}=-\frac{1}{2} \chi\left(\partial Z^{-}\right)
$$

Since there is only one exiting boundary component of genus $g^{-}$we have that

$$
2=2-2 g^{-} \text {which implies } g^{-}=0 .
$$

Similar computations hold for a sink.

If the basic set in $Z$ is an index 2 singularity, the Poincaré-Hopf formula implies that

$$
\begin{gathered}
(-1)^{2}=\sum_{j=1}^{e^{+}}\left(1-g_{j}^{+}\right)-\sum_{i=1}^{e^{-}}\left(1-g_{i}^{-}\right), \quad i . e ., \\
\sum_{i=1}^{e^{-}} g_{i}^{-}-\sum_{j=1}^{e^{+}} g_{j}^{+}=1+e^{-}-e^{+} .
\end{gathered}
$$


In conjunction with the previous bounds we have obtained for the degree of $v$, the above expression reduces to the following two cases:

(a) $g_{1}^{-}=g_{1}^{+}+g_{2}^{+}$when $e^{+}=2$ and $e^{-}=1$;

(b) $g_{1}^{-}=1+g_{1}^{+}$when $e^{+}=e^{-}=1$.

Similar computations hold for an index one singularity.

Let the basic set in $Z$ be an attracting periodic orbit. Hence, $Z$ has only one entering boundary component. Since there are no singularities in $Z, \sum I_{p}=0$. Thus, the Poincaré-Hopf formula reduces to $0=\frac{1}{2} \chi\left(\partial Z^{+}\right)$. This implies that $g^{+}$ must be equal to one.

Now, let $Z$ contain a one-dimensional basic set which is either the suspension of a subshift of finite type or a saddle type periodic orbit. As before, we have that $\sum I_{p}=0$. It is readily seen that the Poincaré-Hopf formula implies that $\sum_{i=1}^{e^{-}} g_{i}^{-}-\sum_{j=1}^{e^{+}} g_{j}^{+}=e^{-}-e^{+}$.

5. Basic blocks. Given an abstract Lyapunov graph $L$ that satisfies conditions 1, 2 and 3 of Theorem 3.1 we wish to produce a Smale flow on $S^{3}$ with an associated Lyapunov function that has a Lyapunov graph $L^{\prime}$ topologically equivalent to $L$.

The scheme will be to realize the dynamics that each vertex $v$ is labelled with, on a submanifold of $S^{3}$ with boundary. The submanifold $X$ must possess only one basic set, namely the one that $v$ is labelled with. Also, the flow will enter (exit) $X$ through $e^{+}\left(e^{-}\right)$boundary components $\left\{\partial X_{j}^{+}\right\}_{j=1}^{e^{+}}\left(\left\{\partial X_{i}^{-}\right\}_{i=1}^{e^{-}}\right)$of $X$, where $e^{+}$ $\left(e^{-}\right)$is the number of incoming (outgoing) edges of $v$. Furthermore, each boundary component $\partial X_{j}^{+}\left(\partial X_{i}^{-}\right)$which is a 2-manifold must have genus $g_{j}^{+}\left(g_{i}^{-}\right)$where $g_{j}^{+}$ $\left(g_{i}^{-}\right)$are the weights on the incoming (outgoing) edges of $v$. Ultimately, we will glue all boundary components of the submanifolds of $S^{3}$ that we will have constructed obeying the orientations prescribed by the graph. The gluing must be done so that we end up with $S^{3}$ and furthermore with a smooth flow defined on it.

Let $H=\bigcup h_{i}$ be a finite set of disjoint 1-handles, i.e., each $h_{i}$ is an embedded copy of $D^{1} \times D^{1}$, where $D^{1}=[-1,1]$. If $x$ is a point of $D^{1} \times p \subset h_{i}, p \in D^{1}$, denote the interval $D^{1} \times p$ by $W_{i}^{u}(x)$. If $x \in q \times D^{1} \subset h_{i}, q \in D^{1}$, denote $q \in D^{1}$ by $W_{i}^{s}(x)$.

DEFINITION 5.1. The diffeomorphism $f$ is hyperbolic with respect to the set of handles $H$ provided

(1) if $x \in h_{i}, f(x) \in h_{j}$ then int $f\left(W_{i}^{u}(x)\right) \supset W_{j}^{u}(f(x))$ and $f\left(W_{i}^{s}(x)\right) \subset$ $\operatorname{int} W_{j}^{s}(f(x))$ and

(2) if $x \in h_{i}, f(x) \in H$ and $v \in T_{x}\left(W_{i}^{s}(x)\right), w \in T_{x}\left(W_{i}^{u}(x)\right)$ then

$$
\begin{gathered}
\|d f(v)\| \leq \lambda\|v\| \quad \text { and } \\
\|d f(w)\| \geq \lambda^{-1}\|w\| \text { for some } \lambda \in(0,1)
\end{gathered}
$$

where \|\| is the standard metric on $D^{1} \times D^{1}$.

From here one can see that $\Lambda=\bigcap_{n \in Z} f^{n}(H)$ has a hyperbolic structure and that if $x \in \Lambda$ then $W_{i}^{u}(x) \subset W^{u}(x)$ and $W_{i}^{s}(x) \subset W^{s}(x)$.

We refer to $H$ as a hyperbolic handle set.

DEFINITION 5.2. The geometric intersection matrix $G$ corresponding to $f$ and the hyperbolic handle set $H$ is defined by $G_{i j}=$ number of components of $h_{i} \cap f\left(h_{j}\right)$ (equivalently we can define $G_{i j}=$ number of points of intersection of $W_{i}^{s}(y)$ with $f\left(W_{j}^{u}(x)\right)$ for any $\left.y \in h_{i}, x \in h_{j}\right)$. 
We will now proceed to construct these submanifolds of $S^{3}$ which we will refer to as basic blocks [8].

DEFINITION 5.3. A basic block for a one-dimensional basic set $\Lambda$ of a Smale flow $\phi$ on $M$ and a Lyapunov function $g: M \rightarrow \mathbf{R}$ with $g(\Lambda)=c$ is the component of $X=g^{-1}([c-\varepsilon, c+\varepsilon])$ which contains $\Lambda$, where $\varepsilon>0$ is chosen so that $X$ contains no other basic set and

(1) There exists (not necessarily connected) codimension one submanifolds with boundary $U$ and $V$ in $X$ with $U \subset V$, where $V$ is everywhere transverse to the flow.

(2) The first return map $r: U \rightarrow$ int $V$ is a well defined smooth map and there is a hyperbolic handle set $H \subset$ int $U$ with every orbit of $\Lambda$ intersecting $H$ and every $h_{i} \subset H$ intersecting $\Lambda$.

(3) If $x \in H$ but $r(x) \notin H$ then $\phi_{t}(x) \cap H=\varnothing \forall t>0$ and $g\left(\phi_{t_{0}}(x)\right)=c-\varepsilon$ for some $t_{0}>0$. Likewise, if $x \in H$ but $r^{-1}(x) \notin H$ then $\phi_{t}(x) \cap H=\varnothing \forall t<0$ and $g\left(\phi_{t_{0}}(x)\right)=c+\varepsilon$ for some $t_{0}<0$.

A basic block for a zero-dimensional basic set is a submanifold of $S^{3}$ with boundary which will be required to contain the singularity and no other chain recurrent point. Furthermore the flow must be transverse to the boundary.

Our goal is to construct a basic block realizing the dynamics with which the vertex $v$ is labelled. The number of incoming (outgoing) edges of $v$ will be the number of boundary components of the basic block in which the flow enters (exits). Furthermore the genera of the boundary components correspond to the weights on the incoming (outgoing) edges of $v$.

6. Constructing basic blocks for singularities. Given an abstract Lyapunov graph $L$ with a vertex $v$ labelled with a singularity of index zero (a sink), condition 1 of Theorem 3.1 implies that there is only one incoming edge on $v$, i.e., $e^{+}=1$ and condition 3 implies that its weight $g^{+}$is zero. Therefore, we would like to construct a basic block $X$ for a sink which has one boundary component of genus zero. The obvious choice for $X$ is the 3 -ball (or 3-disk) $D^{3}$ of $\mathbf{R}^{3}$ defined by $\|x\| \leq 1$ where \|\| is the euclidean norm. The sink is placed in the geometric center of $D^{3}$ and the flow will be pointing transversely inward on the boundary which is homeomorphic to $S^{2}$. The construction of a basic block for a singularity of index 3 (a source) is obtained by reversing the flow on the basic block for a sink.

If the vertex $v$ is labelled with a singularity of index 2 , condition 2 of the theorem asserts that there are two possibilities for the degrees of $v$ :

(a) $e^{+}=2$ and $e^{-}=1$ or

(b) $e^{+}=e^{-}=1$.

The Poincaré-Hopf condition implies in case (a) that if the incoming edges have weights $p$ and $q$, the outgoing edge must have weight $p+q$. In case (b) if the incoming edge has weight $p$, the outgoing edge must have weight $p+1$.

In order to construct the two possible types of basic blocks for the singularity of index 2, we will first consider a flow on a solid cylinder $C=D^{2} \times[0,1]$ as follows: place the index 2 singularity in the geometric center of $C$ in such a way that the flow will enter the cylinder $C$ through the two boundary components homeomorphic to $D^{2}$ and will exit elsewhere as in Figure 6.1.

To construct a basic block satisfying the requirements in case (a), consider two handlebodies $M_{1}$ and $M_{2}$ of genus $p$ and $q$ respectively. Let $\mathbf{M}_{1}$ and $\mathbf{M}_{2}$ denote the respective collarings of $M_{1}$ and $M_{2}$. Consider the flow on the collars $\mathbf{M}_{i}-M_{i}$, 


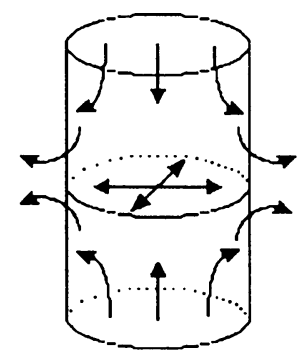

FIGURE 6.1

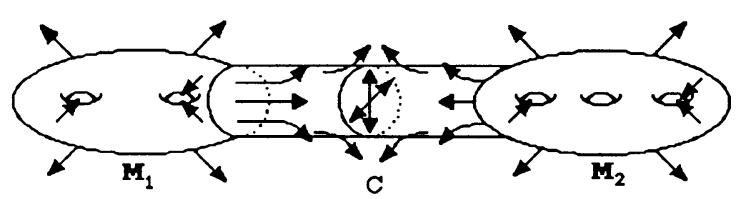

FIGURE 6.2

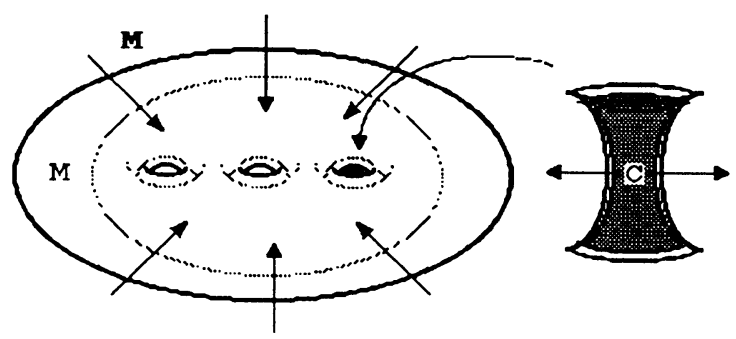

FIGURE 6.3

$i=1,2$, as defined previously. Since $\mathbf{M}_{1}$ and $\mathbf{M}_{2}$ are disjoint, glue the solid cylinder $C$ so that one of the ends homeomorphic to $D^{2}$ is glued on $\partial \mathbf{M}_{1}$ and the other on $\partial \mathbf{M}_{2}$. The basic block will be $X=\left(\mathbf{M}_{2}-M_{2}\right) \cup C \cup\left(\mathbf{M}_{1}-M_{1}\right)$. The two boundary components of $X$ where the flow enters will be 2-manifolds of genus $p$ and $q\left(\partial M_{1}\right.$ and $\partial M_{2}$ respectively). The boundary component of $X$ where the flow exits is a 2-manifold of genus $p+q$ namely $\partial\left(\mathbf{M}_{1} \cup C \cup \mathbf{M}_{2}\right)$. One should round off the corners of $\mathbf{M}_{1} \cup C \cup \mathbf{M}_{\mathbf{2}}$ to obtain a smooth manifold. See Figure 6.2.

To construct the basic block satisfying the requirements in case (b) consider a handlebody $M$ of genus $p+1$ and its collaring $\mathbf{M}$. Consider a flow on $\mathbf{M}-M$ as defined previously. Let $\alpha$ be a nonseparating curve on $\partial \mathbf{M}$ and $A$ be an annulus on $\partial \mathbf{M}$ containing $\alpha$. Glue onto $A$ the boundary component of the cylinder $C$ where the flow exits. The basic block will be $X=(\mathbf{M}-M) \cup C$. The flow will enter $X$ through a 2-manifold of genus $p$ namely $\partial(\mathbf{M} \cup C)$ and will exit through a 2-manifold of genus $p+1$ which is $\partial M$. In order to construct the two possible types of basic blocks for singularities of index 1 reverse the flow on the basic blocks constructed above. See Figure 6.3. 
7. Constructing basic blocks for periodic orbits If $v$ is a vertex of an abstract Lyapunov graph $L$ labelled with an index zero periodic orbit (i.e., attracting periodic orbit) then condition 1 of the theorem asserts that there is exactly one incoming edge on $v$. The Poincaré-Hopf condition implies that the weight on this edge is equal to one. Our choice of basic block $X$ must have one boundary component of genus equal to one. The obvious choice for $X$ is a solid torus $D^{2} \times S^{1}$ containing an attracting periodic orbit with the flow pointing inwardly transverse to $\partial X$. A basic block for an index 2 periodic orbit (i.e., repelling periodic orbit) can be obtained by reversing the flow on the basic block defined above.

Let the vertex $v$ of the Lyapunov graph be labelled with an index 1 (i.e., saddle type periodic orbit). By condition 2 of the theorem there are four possibilities for the degrees of $v$ :

(a) $e^{+}=e^{-}=2$,

(b) $e^{+}=e^{-}=1$,

(c) $e^{+}=1$ and $e^{-}=2$,

(d) $e^{+}=2$ and $e^{-}=1$.

The Poincaré-Hopf condition implies in cases (a) and (b) that the sum of the weights on the incoming edges must equal the sum of the weights on the outgoing edges. In case (c) it implies that the weight on the incoming edge is one less than the sum of the weights on the outgoing edges. Case (d) is entirely symmetric to case $(c)$.

In order to realize these basic blocks we will make use of the round handles defined in 2.8 .

In case (a) the basic block must have two boundary components of genus $r$ and $s$. Also it must have two exiting boundary components of genus $p$ and $q$, where $r+s=p+q$. Use a connecting invariant round handle $R$ on two collared handlebodies $\mathbf{M}_{2}$ and $\mathbf{M}_{1}$ of genus $p$ and $q$ respectively. Attach the handle so that the flow enters on two handlebodies of genus $r$ and $s$. The basic block is defined as $X=\left(\mathbf{M}_{2}-M_{2}\right) \cup R \cup\left(\mathbf{M}_{1}-M_{1}\right)$.

In case (b) the basic block must have one entering and one exiting boundary component. By the Poincaré-Hopf formula, both should have the same genus $p$. In this case it suffices to attach an invariant round handle $R$ to a collared handlebody $\mathbf{M}$ of genus $p$.

In case (c) the basic block must have one entering and two exiting boundary components. The two exiting boundary components have genus $p$ and $q$. By the Poincaré-Hopf formula the entering boundary component should have genus $p+$ $q-1$. To realize this basic block, attach a decreasing round handle $R$ to two collared handlebodies $\mathbf{M}_{1}$ and $\mathbf{M}_{2}$ of genus $p$ and $q$. The basic block is defined as $X=\left(\mathbf{M}_{2}-M_{2}\right) \cup R \cup\left(\mathbf{M}_{1}-M_{1}\right)$.

8. Constructing basic blocks for suspensions of subshifts of finite type $\sigma(A)$. By Bowen's theorem a vertex in $L$ representing a one-dimensional basic set which is not a periodic orbit can be labelled with the suspension of the subshift of finite type $\sigma(A)$. For simplicity we label the vertex $v$ with the matrix $A$ that represents the subshift. Let $e^{+}$and $e^{-}$be the indegree and outdegree of $v$, respectively, which satisfies condition 2 of Theorem 3.1. Let $\left\{g_{j}^{+}\right\}$and $\left\{g_{i}^{-}\right\}$be the weights on the incoming and outgoing edges of $v$, respectively, satisfying the Poincaré-Hopf condition. 
Our aim is to construct a basic block for the suspension of $\sigma(A)$. Alternatively, we can construct a basic block for the suspension of $\sigma(N)$ where $N$ is a matrix that is flow equivalent to $A$. By definition, this implies that the suspensions of $\sigma(A)$ and $\sigma(N)$ are topologically equivalent. An essential proposition at this point is due to Franks [8].

PROPOSITION 8.1. If $A$ is a nonnegative irreducible integer matrix which is not a permutation matrix then given an integer $M>0$ there is a matrix $N$ which is flow equivalent to $A$ and which has every entry greater than $M$ and nondiagonal entries even. The size of $N$ depends only on $A$ and not on $M$.

By this proposition, the matrix $A^{m \times m}$ is flow equivalent to some matrix $N^{n \times n}$ which has all nondiagonal entries even. Furthermore, $\operatorname{dim} \operatorname{ker}\left((I-\tilde{N}): F_{2}^{n} \rightarrow F_{2}^{n}\right)=$ $\operatorname{dim} \operatorname{ker}\left((I-\tilde{A}): F_{2}^{m} \rightarrow F_{2}^{m}\right)=k$ since this is an invariant of flow equivalence [4], where $\tilde{N}$ denotes the mod 2 reduction of $N$.

The construction of the basic block will break up into two parts, A and B. In part A we will prove Proposition 8.2 which constructs a basic block for a vertex $v^{\prime}$ labelled with the matrix $\tilde{N}$. The vertex $v^{\prime}$ will have the same indegree and outdegree as the vertex $v$. The weights on the incoming and outgoing edges of $v^{\prime}$ will be the same as those on $v$. We remark that this proposition will construct geometrically the basic block sought for $v$. In other words the basic block will have the desired number of entering and exiting boundary components $\left(e^{+}\right.$and $\left.e^{-}\right)$with the appropriate genera that corresponds to the given weights. The only drawback is that the dynamics in the basic block is that of the suspension of $\sigma(\tilde{N})$ and not of $\sigma(N)$. In part B we will overcome this problem by isotoping one-handles (see Definition 8.6) of the round handles within flow boxes of small diameter, so that the basic block will then represent the dynamics of the suspension of $\sigma(N)$.

A. Construction of a basic block for the suspension of $\sigma(\tilde{N})$.

PROPOSITION 8.2. Let $v$ be a vertex of L labelled with the suspension of $a$ subshift of finite type $\sigma(\tilde{N})$, where $\tilde{N}^{n \times n}$ is a matrix with $k$ ones on the diagonal and all other entries zero. Let $e^{+}$and $e^{-}$be the indegree and outdegree of $v$ and $\left\{g_{j}^{+}\right\}$and $\left\{g_{i}^{-}\right\}$be the weights on the incoming and outgoing edges of $v$ respectively. Then there exists a basic block $X$ for the suspension of $\sigma(\tilde{N})$ with $e^{+}\left(e^{-}\right)$entering (exiting) boundary components each being a 2-manifold of genus $g_{j}^{+}\left(g_{i}^{-}\right)$.

Since the dynamics of the suspension of $\sigma(\tilde{N})$ is fairly simple, namely, it consists of a basic set with $k$ periodic orbits, the difficulty at this point is to be able to realize geometrically a set which has the appropriate entering and exiting boundary components specified by the graph. The idea used here will be similar to the construction of basic blocks for saddle type periodic orbits. We will glue $k$ round handles onto collared handlebodies and the basic block $X$ will consist of the collarings together with the round handles.

DEFINITION 8.3. A pair of handlebodies $\left(M_{i}, M_{j}\right)$ is distinct from a pair $\left(M_{l}, M_{k}\right)$ if $\{i, j\} \neq\{l, k\}$.

To prove Proposition 8.2 we will use the following lemma:

LEMMA 8.4. Let $e^{+}$and $e^{-}$be positive integers such that $e^{-} \geq e^{+}$. Let $\left\{g_{j}^{+}\right\}$, $j=1, \ldots, e^{+}$, and $\left\{g_{i}^{-}\right\}, i=1, \ldots, e^{-}$, be collections of nonnegative integers (not 
all simultaneously zero) such that $e^{-}-e^{+}=\sum g_{i}^{-}-\sum g_{j}^{+}$. Consider $e^{-}$disjoint collared handlebodies $\mathbf{M}_{i}$ embedded in $S^{3}$ each of genus $g_{i}^{-}$. Let $V=\left(S^{3}-\bigcup_{i=1}^{e^{-}} \mathbf{M}_{i}\right)$. Then there are $e^{-}-1$ round handles that disconnect $V$ into $e^{+}$disjoint handlebodies of genera $g_{j}^{+}$.

By reversing the roles of $e^{+}$and $e^{-}$and reversing the flow on the round handles, the above lemma takes care of the case of $e^{+} \geq e^{-}$.

Lemma 8.4 is a geometric procedure for gluing $e^{-}-1$ round handles (connecting invariant and connecting decreasing round handles) to $e^{-}$collared handlebodies. If we consider the collars and the round handles with the flow defined as before, we obtain a basic block with $e^{+}+e^{-}$boundary components with the appropriate genera. At this point this basic block does not realize $\sigma(\tilde{N})$. It realizes the suspension of a subshift of finite type given by the $\left(e^{-}-1\right) \times\left(e^{-}-1\right)$ identity matrix.

We will postpone the proof of Lemma 8.4 and proceed with the proof of Proposition 8.2.

PROOF (PROPOSITION 8.2). To realize the suspension of $\sigma(\tilde{N})$ we will proceed as in Lemma 8.4 i.e., $e^{-}-1$ connecting round handles will be glued onto $e^{-}$collared handlebodies $M_{i}$ of genera $g_{i}^{-}$. At this point, Lemma 8.4 has constructed a basic block $X^{\prime}$ for a basic set which consists of $e^{-}-1$ periodic orbits. To account for the $k$ periodic orbits, $k-\left(e^{-}-1\right)$ invariant round handles must be glued onto $X^{\prime}$. These $k$ periodic orbits will correspond to the $k$ ones on the diagonal of $\tilde{N}$. Also, $n-k$ nilpotent handles must be glued onto $X^{\prime}$. Recall that these handles are homeomorphic to $D^{1} \times D^{1} \times D^{1}$ and contain no chain recurrent points. These handles will account for the $n-k$ diagonal zeroes of $\tilde{N}$. Denote this modified $X^{\prime}$ by $X$. Note that $e^{+}, e^{-},\left\{g_{j}^{+}\right\}$and $\left\{g_{i}^{-}\right\}$have remained the same for $X$ i.e., adding invariant handles and nilpotent handles to $X^{\prime}$ does not alter the number of boundary components of $X^{\prime}$ or their genera. Q.E.D.

PROOF (LEMMA 8.4). The scheme will be to use $e^{+}-1$ connecting invariant round handles $R_{j}$ and $e^{-}-e^{+}$connecting decreasing handles $D_{k}$ such that:

(1) the $D_{k}$ 's connect distinct pairs of $\mathbf{M}_{i}$ 's forming a new collection of $e^{+}$handlebodies $\left\{N_{j}\right\}$;

(2) the $R_{j}$ 's connect distinct pairs of $N_{j}$ 's;

(3) the $\partial\left(\bigcup\left(N_{j} \cup R_{j}\right)\right)$ consists of $e^{+}$components, each a surface of genus $g_{j}^{+}$, $j=1, \ldots, e^{+}$.

Let us reorder the sets $\left\{g_{j}^{+}\right\}$and $\left\{g_{i}^{-}\right\}$to be in decreasing order. We will first consider the case where $e^{+}$and $e^{-}$are equal. The method used will be multiple induction on the number $L=e^{-}$of collared handlebodies and the sum $G=\sum g_{i}^{-}$ of the genera of the $M_{i}$ for $i=1, \ldots, L$.

For $L=1$ and $G=1$ the lemma is trivially satisfied. Also if we assume $L=1$ with genus $G$ to be true it is equally easy to see that $L=1$ with genus $G+1$ is true.

Next as our inductive hypothesis we will assume the case where there are $L$ collared handlebodies with the sum of their genera being $G$ to be true. We wish to prove that the case where there are $L+1$ collared handlebodies with the sum of their genera being $G$ is true.

Given $g_{1}^{+} \geq g_{2}^{+} \geq \cdots \geq g_{L+1}^{+}$and $g_{1}^{-} \geq g_{2}^{-} \geq \cdots \geq g_{L+1}^{-}$choose $L$ collared handlebodies each of genus $g_{i}^{-}$for $i=1, \ldots, L$. 
CLAIM 1. $g_{L+1}^{+}+\cdots+g_{3}^{+}<g_{1}^{-}+\cdots+g_{L}^{-}$.

PROOF. By hypothesis $g_{L+1}^{+}+\cdots+g_{3}^{+}=g_{1}^{-}+\cdots+g_{L}^{-}+g_{L+1}^{-}-g_{2}^{+}-g_{1}^{+}$. So it suffices to prove that

$$
g_{L+1}^{-}<g_{2}^{+}+g_{1}^{+}
$$

Suppose that (1) is not true. Since the collection $\left\{g_{i}^{+}\right\}$is in decreasing order and $g_{1}^{+}>0$ this would imply that

$$
g_{i}^{+}<g_{L+1}^{-} \forall i=2, \ldots, L+1 \text {. }
$$

Inequality (2) implies that $g_{1}^{+}+\cdots+g_{L}^{+}+g_{L+1}^{+}<g_{1}^{-}+\cdots+g_{L}^{-}+g_{L+1}^{-}$which contradicts the hypothesis that guarantees equality. Q.E.D. (Claim 1)

By Claim 1 there exists a positive integer $g^{*}>0$ such that

$$
g_{L+1}^{+}+\cdots+g_{3}^{+}+g^{*}=g_{1}^{-}+\cdots+g_{L}^{-} \text {. }
$$

We know that $g_{L+1}^{+}+\cdots+g_{3}^{+}+g^{*}=g_{1}^{-}+\cdots+g_{L}^{-}$. Given $L$ collared handlebodies $\mathbf{M}_{i}$ of genus $g_{i}^{-}, i=1, \ldots, L$, by our induction hypothesis we can glue $L-1$ round handles $R_{j}$ onto $\partial \mathbf{M}_{i}$ so that $\partial\left(\left(\bigcup \mathbf{M}_{i}\right) \cup\left(\bigcup R_{j}\right)\right)$ consists of $L$ components, each component being a surface of genus $g^{*}$ or $g_{k}^{+}$for $k=3, \ldots, L+1$. Each of these surfaces bounds a handlebody of genus $g_{k}^{+}$and one of genus $g^{*}$. Note that these handlebodies are disjoint and do not intersect with $M_{i}, i=1, \ldots, L$, although their boundaries intersect with the boundaries of the collared handlebodies $\mathbf{M}_{i}$.

At this point we wish to introduce the collared handlebody $\mathbf{M}_{L+1}$ of genus $g_{L+1}^{-}$ into our construction. We also wish to change the genus $g^{*}$ of $M^{*}$, to $g_{1}^{+}$and $g_{2}^{+}$. In order to do this we must embed $M_{L+1}$ in the interior of $M^{*}$ to enable us to glue a connecting invariant round handle $R_{L}$ from $\partial \mathbf{M}_{L+1}$ to the annulus $A \subset \partial \mathbf{M}_{i} \cap \partial M^{*}$ for some $i=1, \ldots, L$. Recall that $g^{*}+g_{L+1}^{-}=g_{1}^{+}+g_{2}^{+}$. This implies that the connecting invariant round handle $R_{L}$ can be glued onto $\partial \mathbf{M}_{L+1}$ and $\partial M^{*}$ so that the $\partial\left(\mathbf{M}_{L+1} \cup R_{L} \cup M^{*}\right)$ consists of two components, each a surface of genus $g_{1}^{+}$ and $g_{2}^{+}$.

In the case where $e^{-}>e^{+}$the problem is reduced to the previous case in the following way. We will first glue $e^{-}-e^{+}$decreasing connecting round handles $D_{k}$ 's onto distinct pairs of $\partial \mathbf{M}_{i}$ 's where $i=1, \ldots, L+1$ forming a new collection of $e^{+}$handlebodies $N_{j}, j=1, \ldots, e^{+}$. The sum of the genera of the $N_{j}$ 's will be $\sum g_{i}^{-}-\left(e^{-}-e^{+}\right)$which is equal to $\sum g_{j}^{+}$. So we are in the previous case where $e^{+}=e^{-}$. Therefore we can glue $e^{+}-1$ connecting invariant round handles $R_{s}$ onto the $\partial N_{j}$ 's so that the $\partial\left(\left(\cup N_{j}\right) \cup\left(\bigcup R_{s}\right)\right)$ consists of $e^{+}$components, each being a surface of genus $g_{j}^{+}, j=1, \ldots, e^{+}$. Q.E.D.

Before we proceed in the construction of the basic block for $\sigma(N)$ let us describe a procedure to label the round handles.

Proposition 8.2 has given us a procedure for constructing a basic block for a suspension of a subshift of finite type. Recall that we attach $k$ round handles to $e^{-}$collared handlebodies $\mathbf{M}_{i}, i=1, \ldots, e^{-}$. We can assume the handlebodies to be in a linear order. For simplicity we will assume that the decreasing round handles in Lemma 8.4 connect distinct consecutive pairs of $\mathbf{M}_{i}$ 's. The linear order of the handlebodies $\mathbf{M}_{i}$ and the fact that a connecting decreasing round handle or a connecting invariant round handle are always attached to two distinct handlebodies 


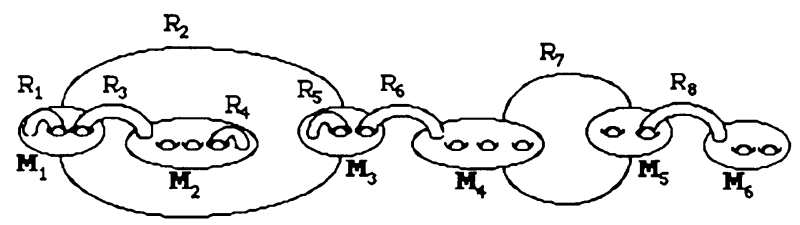

FIGURE 8.1

enables us to distinguish their left-hand end and right-hand end. If a connecting decreasing handle or a connecting invariant handle is glued onto $\partial \mathbf{M}_{s}$ and $\partial \mathbf{M}_{t}$ and $s<t$ then the left-hand end will be the end glued onto $\partial \mathbf{M}_{s}$ and the right-hand end to $\partial \mathbf{M}_{t}$. Since an invariant handle has both its ends glued onto the same collared handlebody the left-hand end can be chosen arbitrarily.

DEFINITION 8.5. Let $R_{i}$ be a connecting invariant round handle that has its left-hand end glued onto $\partial \mathbf{M}_{s}$ and its right-hand end glued onto $\partial \mathbf{M}_{t}$. A round handle $R_{j}$ is locked in $R_{i}$ if its left-hand end is glued onto $\partial \mathbf{M}_{u}, u \geq s$, and its right-hand end is glued onto $\partial \mathbf{M}_{v}, v \leq t$.

In Figure $8.1, R_{3}, R_{4}$ and $R_{5}$ are locked in $R_{2}$. In Figure $8.2 R_{2}, \ldots, R_{7}$ are locked in $R_{1}$. Also $R_{4}, R_{5}, R_{3}$ and $R_{6}$ are locked in $R_{2}$.

Let us describe a systematic way to order the round handles $R_{s}, s=1, \ldots, k$. If there are no locked handles one will label them in linear order from left to right.

If there are locked handles one will use the following scheme to order the round handles. We will label from left to right in increasing order until a round handle $R_{i}$ which possesses $s$ locked handles is reached. Then we label the locked handles in $R_{i}$ from left to right with $R_{i+1}, R_{i+2}, \ldots, R_{i+s}$. The handle which has its lefthand end on the same handlebody as the right-hand end of $R_{i}$ will be labelled with $R_{i+s+1}$. If there is more than one handle with this property they will be locked in a connecting invariant round handle which we will label with $R_{i+s+1}$. We will continue to label the round handles in linear order from left to right until another round handle which possesses locked handles is reached. See Figure 8.1.

Note that there can be a connecting invariant round handle $R_{i}$ which possesses a connecting invariant round handle $R_{j}$ as one of its locked handles, and $R_{j}$ in turn possesses other locked handles. In this case one applies the labelling scheme recursively. See Figure 8.2.

Let us remark that $e^{-}$handlebodies must be connected by $e^{-}-1$ connecting round handles to form one connected component. This implies that a connecting invariant round handle $R_{i}$ which possesses $s$ locked handles $R_{i+1}, \ldots, R_{i+s}$ has the following property: if the left-hand ends of $R_{i}$ and of $R_{i+1}$ are on $\partial \mathbf{M}_{t}$ cind the right-hand ends of $R_{i}$ and of $R_{i+s}$ are on $\partial \mathbf{M}_{r}$ then there exists at least one $w$, $1 \leq w<s$, such that the right-hand end of $R_{w}$ and the left-hand end of $R_{w+1}$ are on distinct components of $\partial \mathbf{M}$. If such a locked round handle $R_{w}$ did not exist we would be using a connecting round handle on a connected component. This would imply that $e^{-}$handlebodies could not be connected by $e^{-}-1$ connecting round handles. The resulting construction would contradict Lemma 8.4 since the round handles would not disconnect $V$ into handlebodies.

B. Realizing the suspension of $\sigma(N)$. Let $N^{n \times n}$ be as in Proposition 8.1. By Proposition 8.2 we are able to construct a basic block $X$ for the suspension of $\sigma(\tilde{N})$, 


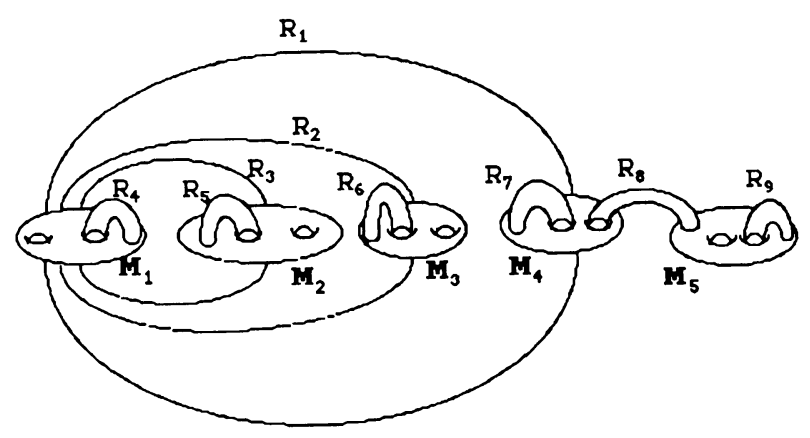

FIGURE 8.2

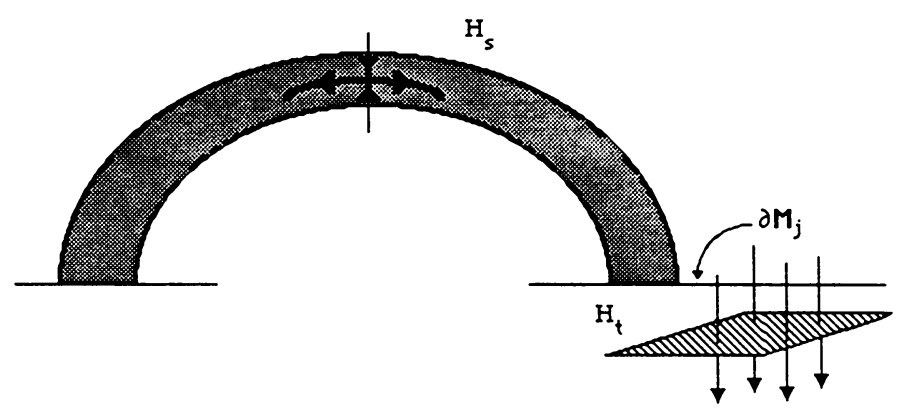

FIGURE 8.3

where $\tilde{N}$ is the mod 2 reduction of $N$. Our goal is to construct a basic block for the suspension of $\sigma(N)$ or for the suspension of $\sigma(E)$ where $N$ and $E$ are flow equivalent. In order to achieve this, we wish to maintain the basic block $X$ while modifying the flow within it.

Let us define one-handles, $H_{i}$ of round handles and nilpotent handles.

DEFINITION 8.6. A one-handle $H_{i}$ is homeomorphic to a strip $D^{1} \times D^{1}$ and is transversal to the flow. A one-handle $H_{s}$ in a round handle $R_{s}$ will be chosen so that $H_{s} \times S^{1}=R_{s}$. A one-handle $H_{t}$ in a nilpotent handle $N_{t}$ will be chosen se that $H_{t} \times D^{1}=N_{t}$ and $\phi_{-\eta}\left(H_{t}\right) \subset \partial \mathbf{M}_{j}$ for some $\eta>0$ and some $j>0$. See Figure 8.3.

Note that $\eta>0$ exists since $N_{t}$ is embedded in the collar of $\partial M_{j}$ for some $j>0$.

We will assume that the one-handles $H_{s}, s=1, \ldots, k$, are one-handles in round handles $R_{s}$ and that $H_{t}, t=k+1, \ldots, n$, are one-handles in a nilpotent handle $N_{t}$. Let $x$ be a point of $D^{1} \times p \subset H_{i}$ where $p \in D^{1}$. The interval $D^{1} \times p$ will be denoted $W_{i}^{u}(x)$. Similarly if $x$ is a point of $q \times D^{1}$ the interval $q \times D^{1}$ will be denoted by $W_{i}^{s}(x)$.

The reason we introduce one-handles is that if $H_{i}$ intersects $r\left(H_{j}\right) m$ times then the $i j$ th entry of $\tilde{N}$ increases by $m$, where $r(x)=\phi_{1}(x)$ is the first return map for the one-handle of the round handles. Actually it is sufficient that the stable manifold $W_{i}^{s}(x)$ intersect the unstable manifold $r\left(W_{j}^{u}(y)\right), \forall x \in H_{i}$ and $y \in H_{j}$.

This is precisely what we want to do, increase the entries of $\tilde{N}$ to obtain $N$. 


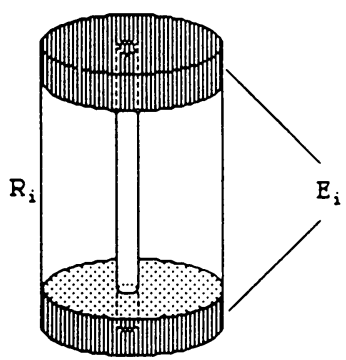

FIGURE 8.4

Unfortunately we do not obtain $N$ but a matrix $E$ which is flow equivalent to it. For our purpose this is sufficient since the suspension flow for $\sigma(N)$ is topologically equivalent to the suspension flow for $\sigma(E)$.

The spirit of the proof from here on will be to push unstable manifolds through stable manifolds to increase the entries of $\tilde{N}$. This pushing will be done within flow boxes defined in $X$.

The proof at this point will break up into the following steps:

(1) Construction of $n$ disjoint flow boxes contained in $X$.

(2) Homotopies will be performed within the flow boxes and the basic block will then realize the suspension of $\sigma(E)$ where $E$ and $N$ are flow equivalent.

(1) Construction of the flow boxes. Let us now focus our attention in the construction of the flow box $F_{j}$. We will then be concerned in showing that the collection $\left\{F_{j}\right\}_{j=1}^{n}$ of flow boxes can be constructed disjointly.

We must define a flow box $F=\left\{\phi_{t}(x): x \in D\right.$ and $\left.0 \leq t \leq T\right\}$ with the property that it goes through all the round handles and nilpotent handles. We will make the meaning of "goes through" very precise as we proceed with the construction.

DEFINITION 8.7. Let $E_{i}$ be the two disjoint components of the round handle $R_{i}$ which are homeomorphic to $R_{i}$ and have the property that $\phi_{1}\left(E_{i}\right) \cap R_{i}=\varnothing$. We will call $E_{i}$ the set of ends of $R_{i}$. See 8.4.

The flow box $F_{j}$ will be defined in the following region:

$$
Y=\bigcup_{s=1}^{k}\left\{\left(R_{s}-\phi_{1}\left(R_{s}\right)\right) \cup\left(E_{s}\right)\right\} \cup\left\{C-\bigcup_{s=1}^{k} \phi_{1}\left(R_{s}\right)\right\}
$$

where $\left\{R_{s}\right\}_{s=1}^{k}$ is the collection of round handles, $E_{s}$ is the set of ends of $R_{s}$ and

$$
C=\bigcup_{i=1}^{e^{-}}\left[\mathbf{M}_{i}-\phi_{1}\left(\mathbf{M}_{i}\right)\right]
$$

See Figure 8.5.

In order to construct the flow box $F$ we must define a disk $D$. To do so we must define subsets of $D$ homeomorphic to $D$ which we will refer to as strips $h_{i}$ and $h_{i}^{*}$. Let $h_{i}$ and $h_{i}^{*}$ be strips in $H_{i} \subset R_{i}$ where $R_{i}$ is a round handle that does not contain the saddle point. These strips extend all the way across $H_{i}$. Furthermore $h_{i}$ does not intersect the unstable manifold of the saddle point and $h_{i} \cap \phi_{1}\left(H_{i}\right)=\varnothing$. The strip $h_{i}^{*}$ intersects the unstable manifold of the saddle point so that $h_{i}^{*} \cap\left(\phi_{1}\left(H_{i}\right)-E_{i}\right)=\varnothing$ and $h_{i}^{*} \cap\left(\phi_{1}\left(H_{i}\right) \cap E_{i}\right) \neq \varnothing$ as shown in Figure 8.6. 


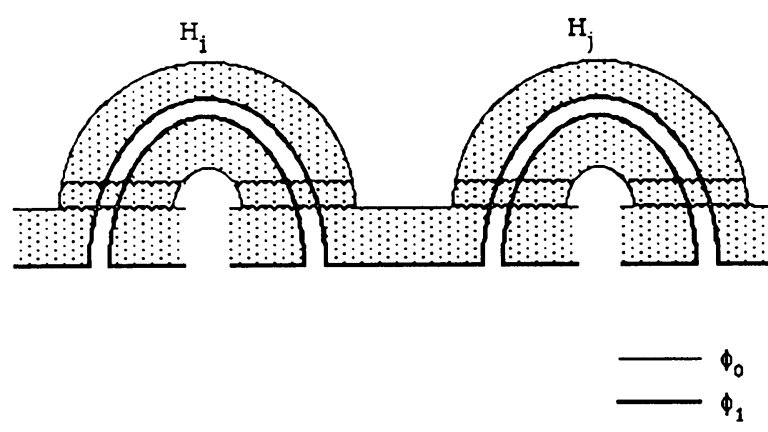

FIGURE 8.5

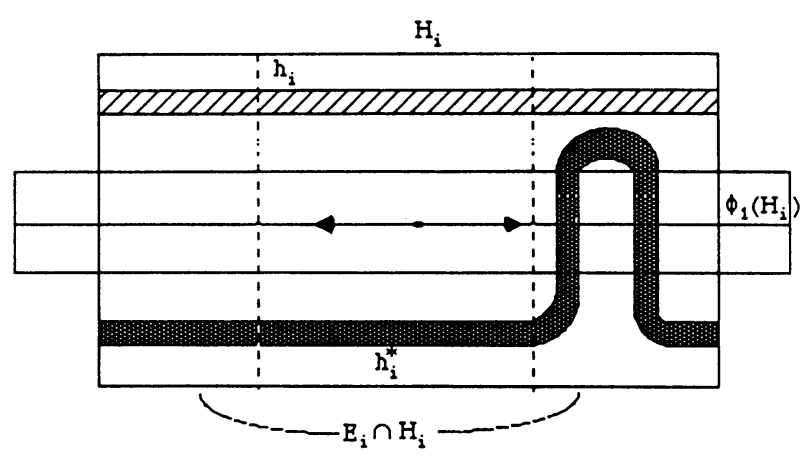

FIGURE 8.6

Our goal is to construct a codimension one disk which goes through all the one-handles of round handles and nilpotent handles. Proposition 8.9 will prove the existence of such a disk which will later be used to define the flow boxes $F_{j}$, $j=1, \ldots, n$. Recall that in defining a round handle $R$ we defined two annuli $A$ and $B$ which were glued onto $\partial \mathbf{M}_{s}$ and $\partial \mathbf{M}_{t}$ respectively for some $s, t>0$. We will define an inner annulus $C$ in $A$ to be an annulus contained in $A$ and concentric with it. Similarly for $B$.

DEFINITION 8.8. Let $\mathbf{M}_{1}, \ldots, \mathbf{M}_{s}$ be collared handlebodies joined by a set of round handles $R$. Let $D$ be a codimension one disk defined in $R \cup \bigcup_{i=1}^{s} \partial \mathbf{M}_{s}$. We say that the disk $D$ has ends on $\partial \mathbf{M}_{1}$ and $\partial \mathbf{M}_{s}$ if there exists two disjoint subsets $e_{1}$ and $e_{2}$ of $D$ defined as follows: $e_{1}$ is the component of $D \cap \partial M_{1}$ which disconnects the disk in precisely two components. Similarly $e_{2}$ is the component of $D \cap \partial M_{s}$ which disconnects the disk in precisely two components.

PROPOSITION 8.9 (Construction of the disk). Given $e^{-}$collared handlebodies with $k$ round handles $R_{i}$ glued onto their boundaries as in Proposition 8.2 define the following region $W$ :

$$
W=\bigcup_{i=1}^{k}\left[\left(H_{i}-\phi_{1}\left(H_{i}\right)\right) \cup\left(E_{i} \cap H_{i}\right)\right] \cup[\partial \mathbf{M}-Z]
$$

where $H_{i}$ are the one-handles of the round handles $R_{i}, E_{i}$ are the ends of the round handles, $\partial \mathbf{M}=\bigcup_{j=1}^{e^{-}} \partial \mathbf{M}_{j}$ and $Z=\bigcup_{i=1}^{2 k} C_{i}$ where $C_{j}$ and $C_{j+k}$ are the inner 


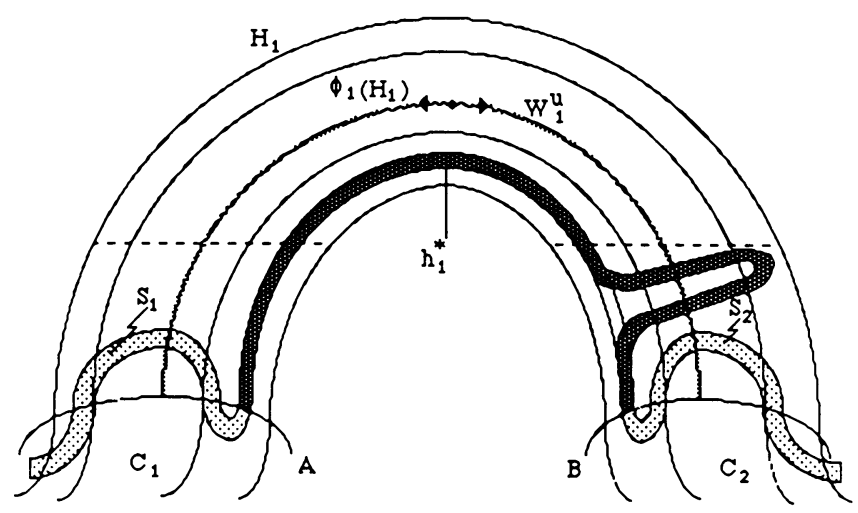

FIGURE 8.7

annuli of the annuli $A_{j}$ and $B_{j}$ of the round handle $R_{j}$. There exists a disk $D \simeq$ $D^{1} \times D^{1}$ in $W$ which has the following properties:

(a) $h_{i}^{*} \subset D \forall i=1, \ldots, k$;

(b) $D \cap H_{i}=h_{i}^{*} \cup h_{i}$ if $R_{i}$ is locked or $D \cap H_{i}=h_{i}^{*}$ otherwise, $\forall i=1, \ldots, k$;

(c) $D$ has ends on $\partial \mathbf{M}_{1}$ and $\partial \mathbf{M}_{e^{-}}$.

PROOF. We will use induction on the number of round handles. Let us start by showing that for one round handle $R$ the disk $D$ exists. Let $R$ be a connecting round handle. We will glue the left-hand of $h_{i}^{*}$ onto a strip $S_{1}$ on $\left(\partial \mathbf{M}_{1}-C_{1}\right) \cup\left(H_{1} \cap E_{1}\right)$ and its right-hand end will be glued onto a strip $S_{2}$ on $\left(\partial \mathbf{M}_{2}-C_{2}\right) \cup\left(H_{1} \cap E_{1}\right)$. See Figure 8.7.

If $R$ is an invariant handle we will glue the left hand of $h_{i}^{*}$ onto a strip $S_{1}$ and its right-hand end will be glued onto a strip $S_{2}$ both defined on $\left(\partial \mathbf{M}_{1}-\left(C_{1} \cup C_{2}\right)\right) \cup$ $\left(H_{1} \cap E_{1}\right)$.

Assume that for $N$ round handles the disk $D$ can be constructed satisfying properties (a), (b) and (c). Let us show that we can construct it for $N+1$ round handles.

Let $R_{1}, \ldots, R_{N+1}$ be the round handles. Remove $R_{1}$ from the basic block $X$. There are two cases to consider:

(1) $R_{1}$ is a connecting round handle (decreasing or invariant);

(2) $R_{1}$ is an invariant round handle.

In case (1) removing $R_{1}$ implies that $X$ will now have two components. One component will consist of $t$ collared handlebodies $M_{1}, \ldots, M_{t}$ connected by round handles. This component possesses altogether $r$ round handles. The other component will consist of $M_{t+1}, \ldots, M_{s}$ and possesses $N-r$ round handles where $1 \leq t<e^{-}$. If $t=1$, then $R_{1}$ is either an invariant connecting round handle or a decreasing round handle. If $1<t<e^{-}, R_{1}$ is an invariant connecting round handle which possesses locked handles. Let us treat the latter case first. By our induction hypothesis there exists a disk $D_{1}$ defined on

$$
W_{1}=\bigcup_{i=2}^{r}\left[\left(H_{i}-\phi_{1}\left(H_{i}\right)\right) \cup\left(E_{i} \cap H_{i}\right)\right] \cup\left[\left(\bigcup_{j=1}^{t} \partial \mathbf{M}_{j}\right)-Z\right] .
$$




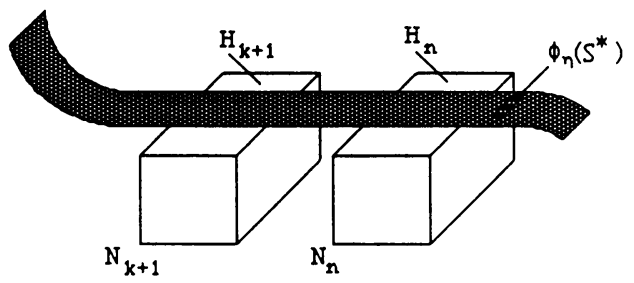

FIGURE 8.8

Also $D_{1} \cap H_{i}=h_{i}^{*}$ since the handles are not locked once we have removed $R_{1}$ and $D_{1}$ has one of its ends on $\partial \mathbf{M}_{1}$ and the other on $\partial \mathbf{M}_{t}$. Similarly, by our induction hypothesis there exists a disk $D_{2}$ defined on

$$
W_{2}=\bigcup_{i=r+1}^{N+1}\left[\left(H_{i}-\phi_{1}\left(H_{i}\right)\right) \cup\left(E_{i} \cap H_{i}\right)\right] \cup\left[\left(\bigcup_{j=t+1}^{e^{-}} \partial \mathbf{M}_{j}\right)-Z\right]
$$

The disk $D_{2}$ has one of its ends of $\partial \mathbf{M}_{t+1}$ and the other on $\partial \mathbf{M}_{e^{-}}$.

Consider the end of $D_{1}$ which is on $\partial \mathbf{M}_{t}$. We will push this end through all handles $H_{j} \subset \bigcup_{i=2}^{r} H_{i}$ which has the property that $R_{j}$ is not locked in some $R_{k}, k=2, \ldots, r$, until it reaches $\partial \mathbf{M}_{1}$. In other words, define a new disk $D_{1}^{*}$ which has the property that $D_{1} \subset D_{1}^{*}$, both ends of $D_{1}^{*}$ are on $\partial \mathbf{M}_{1}$ and $D_{1}^{*}$ contains all $h_{i}, i=2, \ldots, r$.

In order to define $D$ we will glue the left-hand end of $h_{i}^{*}$ to one of the ends of $D_{1}^{*}$ which is on $\partial \mathbf{M}_{1}$. The right-hand end of $h_{i}^{*}$ will be glued onto the end of $D_{2}$ which is on $\partial \mathbf{M}_{t+1}$. $D$ is a disk that satisfies properties a, b and c. If $t=1$ we will define $D$ by connecting the right-hand end of $h_{1}^{*}$ to the end of $D_{2}$ which is on $\partial \mathbf{M}_{2}$. Also the left-hand end of $h_{1}^{*}$ will be glued onto a strip that is on $\left(\partial \mathbf{M}_{1}-C_{1}\right) \cup\left(H_{1} \cap E_{1}\right)$.

In case (2) where $R_{1}$ is an invariant round handle, the basic block $X$ is not disconnected into two disjoint components by removing $R_{1}$. By the induction hypothesis there exists a disk $D_{1}$ defined on

$$
W_{1}=\bigcup_{i=2}^{N+1}\left[\left(H_{i}-\phi_{1}\left(H_{i}\right)\right) \cup\left(E_{i} \cap H_{i}\right)\right] \cup[\partial \mathbf{M}-Z] .
$$

In order to define $D$, one of the ends of $h_{1}^{*}$ will be glued onto the end of $D_{1}$ which is on $\partial \mathbf{M}_{1}$ and the other end of $h_{1}^{*}$ will be glued to a strip that is on $\left(\partial \mathbf{M}_{1}-C_{1}\right) \cup\left(H_{1} \cap E_{1}\right)$. Q.E.D.

Now we will construct $n$ disjoint flow boxes that go through all the round and nilpotent handles.

Let $S^{*}$ be the part of $D$ which intersects $\partial \mathbf{M}_{e^{-}}$. We can place the nilpotent handles in the collar of $M_{e^{-}}$so that $S^{*}$ has the property that $S^{*} \cap \phi_{-\eta} H_{j} \neq \varnothing$ $\forall j=k+1, \ldots, n$. See Figure 8.8.

Let $S^{*} \cap \phi_{-\eta} H_{j}=S_{j}^{*}$. See Figure 8.9. Modify $S_{j}^{*}$ to $S_{j}^{* *} \forall j=k+1, \ldots, n$ as in Figure 8.10. The reason for this is that we want stable and unstable manifolds of each handle foliating the disk. 


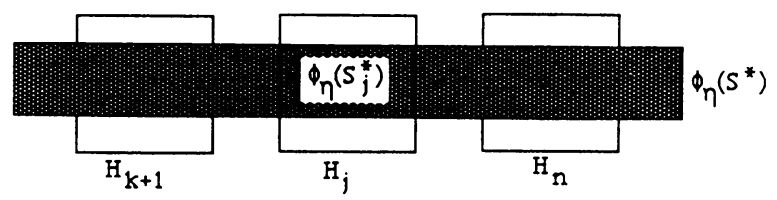

FIGURE 8.9

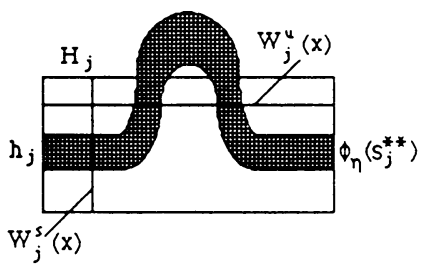

FIGURE 8.10

For simplicity of notation let us continue to denote the modified disk by $D$. Note that the disk $D$ goes through all round and nilpotent handles. Foliate the disk $D$ with $n$ disjoint smaller disks $D_{j}$ which do not intersect and have the same properties as $D$.

Now we can define the flow boxes $F_{j}=\left\{\phi_{t}(x): x \in D_{j}\right.$ and $\left.0 \leq t \leq \eta\right\}$. The flow boxes are also disjoint by construction.

(2) Realizing the suspension of $\sigma(E)$ (where $E$ and $N$ are flow equivalent). Recall that by Proposition 8.2 we were able to realize the suspension of $\sigma(\tilde{N})$ in a basic block $X$ that had the correct number of boundary components with the appropriate genera prescribed by the graph $L$. We have constructed $n$ disjoint flow boxes $F_{j}$ defined in this basic block. We now wish to modify the flow so that the dynamics in the basic block is topologically equivalent to the dynamics with which the vertex $\mathrm{v}$ is labelled. In order to do this we will modify the flow within the flow boxes so that we realize either the suspension of $\sigma(N)$ or the suspension of $\sigma(E)$ where $N$ and $E$ are flow equivalent.

PROPOSITION 8.10. Let $L$ be an abstract Lyapunov graph satisfying the conditions of Theorem 3.1 and $v$ a vertex of $L$ labelled with the suspension of $\sigma(N)$ where $N$ was defined in Proposition 8.1. There exists a basic block $X$ which realizes the suspension of the subshift of finite type $\sigma(E)$, where $E$ and $N$ are flow equivalent.

PROOF. Let $X$ be the basic block constructed in Proposition 8.2 for $\sigma(\tilde{N})$ where $\tilde{N}$ is the mod 2 reduction of $N$. Let $\left\{F_{j}\right\}$ be the collection of $n$ disjoint flow boxes we constructed previously.

The first step is to realize the suspension of $\sigma(C)$ where $C^{n \times n}$ is a matrix that has every entry $c_{i j}=\tilde{n}_{i j}+2 m_{i j}$ where $m_{i j} \in Z^{+}$. We will do this by performing a homotopy in $F_{j}$ that will push the unstable manifold of $H_{j}, \phi_{1}\left(W_{j}^{u}(x)\right)$ through the stable manifolds of all the one-handles $W_{i}^{s}(y)$ transversally. This will increase all $\tilde{n}_{i j}$ entries for $j$ fixed and $i=1, \ldots, n$ by an even number. In this process it will push transversally other unstable manifolds $\phi_{1}\left(W_{s}^{u}(z)\right)$ through stable manifolds of some of the one-handles. This will increase by an even number the $\tilde{n}_{r s}$ entries 

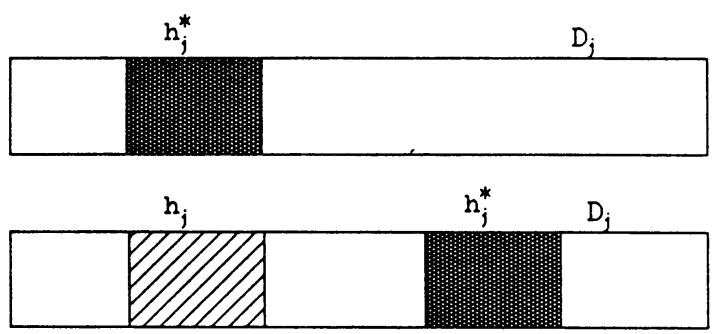

FIGURE 8.11

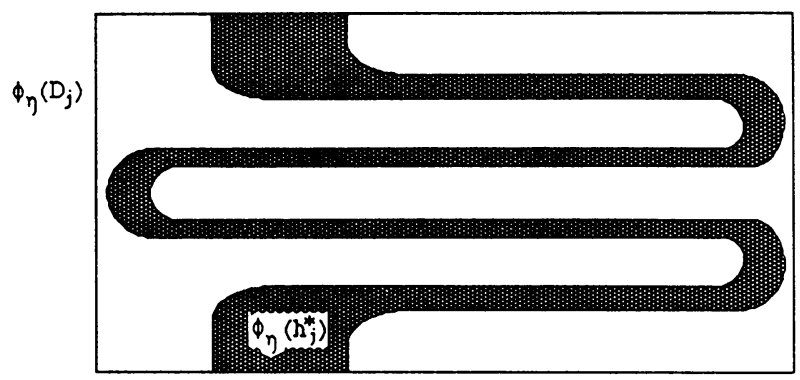

FIGURE 8.12

for some $s>0$ and for some $r>0$. After these homotopies have been performed within the flow boxes, the basic block $X$ realizes the suspension of a subshift of finite type $\sigma(C)$. The matrix $C^{n \times n}$ has entry $c_{i j}=\tilde{n}_{i j}+2 m_{i j}$ where $m_{i j} \in Z^{+}$. This means that $c_{i j} \geq 2 \forall i, j$.

Let us explain this homotopy in more detail. We perform a homotopy on $h_{j}^{*}$. The homotopy will be the identity on a neighborhood of $D_{j}$. The bending of $h_{j}$ and $h_{j}^{*}$ should occur outside the one-handles in order to guarantee transversality. At the end of the homotopy we also want that the set of one-handles be hyperbolic with respect to the first return map $\phi_{1}$. In other words if $\phi_{1}\left(W_{j}^{u}(x)\right) \cap W_{i}^{u}(y) \neq \varnothing$ then $\phi_{1}\left(W_{j}^{u}(x)\right) \supset W_{i}^{u}(y)$ and similarly if $\phi_{1}\left(W_{j}^{s}(x)\right) \cap W_{i}^{s}(y) \neq \varnothing$ then $\phi_{1}\left(W_{j}^{s}(x)\right) \subset$ $W_{i}^{s}(y) \forall x \in H_{j}$ and $y \in H_{i}$.

Depending on the type of the round handle $R_{j}$ there are two possibilities for $H_{j} \cap D_{j}$ :

(a) $H_{j} \cap D_{j}=h_{j} \cup h_{j}^{*}$ or

(b) $H_{j} \cap D_{j}=h_{j}^{*}$. See Figure 8.11.

Whenever $H_{j} \cap D_{j}=h_{j}^{*}$ the homotopy will be as shown. See Figure 8.12. If $H_{j} \cap D_{j}=h_{j}^{*} \cup h_{j}$ the homotopy will be as in Figure 8.13.

Note that after the homotopy $h_{j}^{*}$ intersects all round and nilpotent handles. This means that after the homotopy the unstable manifold of $H_{j}, \phi_{1}\left(W_{j}^{u}(x)\right)$ intersected all stable manifolds of the one-handles $W_{i}^{s}(y)$ transversally.

The homotopy performed on $h_{j}$ of a one-handle in a nilpotent handle $N_{j}$ is the same as in Figure 8.12. 


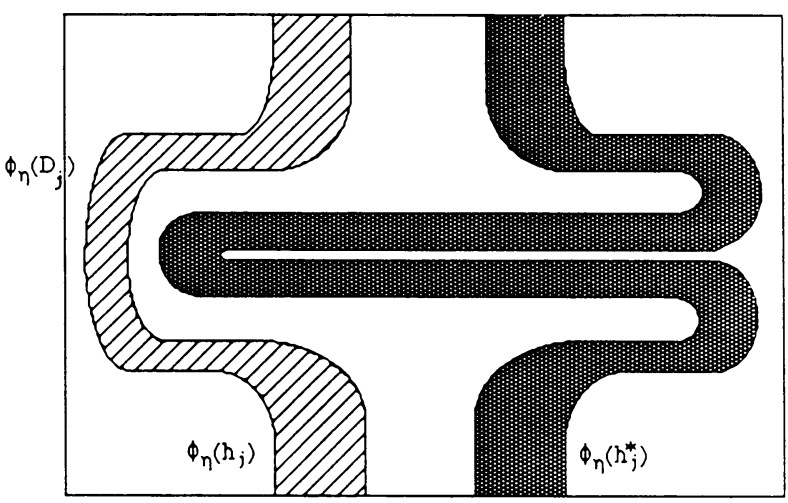

FIGURE 8.13

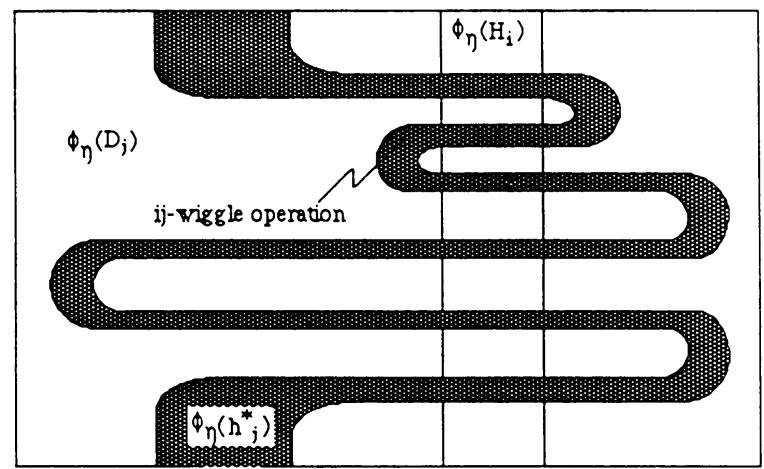

FIGURE 8.14

At this point we would like to gain control over each entry of the matrix $C$, that is increase each $i j$ entry by an even number without altering the other entries. This can be done by performing a wiggle operation in $F_{j}$.

Before we define a wiggle operation, let us assume for simplicity that the homotopy realized in $F_{j}$ is complete after time $\eta / 2$. So for the remaining time $\eta / 2$ the homotopy realized in $F_{j}$ will equal the identity. We will define the $i j$-wiggle operation to be a homotopy performed in $F_{j}$ that will push off a loop from $\phi_{-\eta / 2}\left(h_{j}^{*}\right)$ so that it goes once, only through the round handle $R_{i}$. This $i j$-wiggle operation will increase the $i j$ entry of the matrix exactly by 2. See Figure 8.14.

Another $i j$-wiggle operation can be performed by pushing the loop back through $R_{i}$ again. See Figure 8.15.

Therefore, we can increase the $c_{i j}$ entries of $C$ by performing wiggle operations to form a new matrix $D$ where $d_{i j} \geq n_{i j}$. Let $M=\max \left\{d_{i j}\right\}$. Recall that we were trying to realize $N$. Using Proposition 8.1 once again we obtain that $A^{m \times m}$ is flow equivalent to a matrix $E^{n \times n}$ such that $E$ has all nondiagonal entries even and all entries $>M$. So, it suffies to realize the suspension of $\sigma(E)$. By performing a few more wiggle operations (namely $\frac{1}{2}\left(e_{i j}-d_{i j}\right)$ in $h_{i} \subset F_{j}$ ) we realize the suspension of $\sigma(E)$. Q.E.D. 


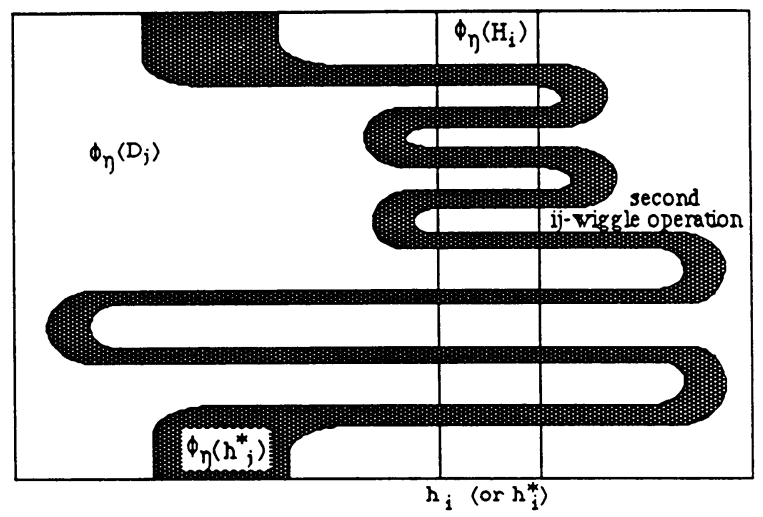

FIGURE 8.15

9. Gluing the basic blocks to obtain a Smale flow on $S^{3}$. So far we have shown that we can realize singularities, periodic orbits and suspensions of subshifts of finite type in basic blocks embedded in $S^{3}$. These basic blocks are submanifolds of $S^{3}$ with boundary.

The final step in proving the main theorem is to glue all the basic blocks we have constructed according to the prescription on the abstract Lyapunov graph $L$ and obtain a Smale flow on $S^{3}$.

Let $X_{v}$ and $X_{w}$ be basic blocks corresponding to the vertices $v$ and $w$ respectively. If there is an edge going from vertex $v$ to vertex $w$ with weight $g$, we will want to glue a component $C^{-}$of $\partial X_{v}^{-}$that has genus equal to $g$ to a component $C^{+}$of $\partial X_{w}^{+}$ that also has genus equal to $g$. Since $C^{-}$and $C^{+}$are unknotted surfaces of genus $g$, they bound in $S^{3}$ unknotted handlebodies on both sides. Let $M_{v}$ (resp. $M_{w}$ ) be the component of the complement of $C^{-}$(resp. $C^{+}$) in $S^{3}$ which contains $X_{v}$ (resp. $\left.X_{w}\right)$. It is helpful to think of these basic blocks as being in different three-spheres. We will glue $M_{v}$ to $M_{w}$ attaching $C^{-}$to $C^{+}$in order to obtain $S^{3}$. If $g^{\prime}$ denotes the attaching map, by Theorem 3.10 in [8] it can be isotoped to $g$ so that the flow on $X=X_{v} \cup_{g} X_{w}$ satisfies the strong transversality condition.

By gluing $M_{v}$ to $M_{w}$ we have formed $S^{3}$ and each boundary component of $X$ is an unknotted surface in $S^{3}$. Choose an edge emanating from the vertex $v$ or $w$ to a new vertex $z$. Repeat the procedure for $X$ and $X_{z}$ producing a new submanifold of $S^{3}$ with its boundary components being unknotted surfaces.

When a source (or sink) vertex $y$ is reached there are two possibilities for $X_{y}$ :

(1) $X_{y}=B^{3}$ the three ball with an index 3 singularity (or an index 0 singularity).

(2) $X_{y}=S^{1} \times D^{2}$, solid torus if $y$ is labelled with a repelling (or an attracting) periodic orbit. We can continue this procedure adding a vertex each time and at each step obtaining a new $X \subset S^{3}$. When all basic blocks $X_{i}$ have been attached for all vertices $i$ then one obtains the three-sphere.

From the Lyapunov function on each $X_{v}$ we can construct a smooth Lyapunov function on $S^{3}$ whose corresponding Lyapunov graph $L^{\prime}$ is topologically equivalent to the abstract Lyapunov graph $L$ with which we started our construction. 
10. Concluding remarks. To see that a Lyapunov graph of a Smale flow on a homology sphere must satisfy the conditions in Theorem 3.1 note that homology spheres have the same homology as the three-sphere. Recall that in the proof of the necessity of these conditions for the three-sphere we only used homology.

It is also interesting to note that one can construct many examples of Smale flows on any closed orientable 3-manifold $M$. Suppose $M$ has a Heegard diagram of genus $g$. Then any abstract Lyapunov graph which is realizable on $S^{3}$ and has at least one edge with weight equal to $g$ is realizable on $M$. Let $e$ be an edge with weight $g$ that connects vertex $v$ to vertex $w$. Let $M_{v}$ be the handlebody of genus $g$ which contains the basic set $X_{v}$ with which the vertex $v$ is labelled. Similarly let $M_{w}$ be the handlebody of genus $g$ containing the basic block $X_{w}$. To obtain $M$ one must identify the boundary of each handlebody following the Heegard instructions. The resulting flow is smooth since on the collar of each handlebody, the vector field tangent to the flow corresponds to $\frac{\partial}{\partial t}$.

An interesting problem would be to determine necessary and sufficient conditions on the Lyapunov graph associated to a Smale flow on 3-manifolds.

Also the same problem for higher-dimensional manifolds is still open.

The question of considering a more general class of flows, for example hyperbolic flows (Axiom A and strong transversality), involves understanding higher dimensional basic sets which at the present moment is at an early stage of development. Studies in this area have been developed by Christy [5].

\section{REFERENCES}

1. D. Asimov, Round handles and nonsingular Morse-Smale flows, Ann. of Math. 102 (1979).

2. B. Bollobas, Graph theory. An introductory course, Springer-Verlag, Berlin, 1979.

3. R. Bowen, One-dimensional hyperbolic sets for flows, J. Differential Equations 12 (1972).

4. R. Bowen and J. Franks, Homology for zero dimensional basic sets, Ann. of Math. 106 (1977).

5. J. Christy, Branched surfaces and attractors I, Dynamic branched surfaces (preprint), Northwestern University.

6. C. Conley, Isolated invariant sets and the Morse index, CBMS Regional Conf. Ser. in Math., vol. 38, Amer. Math. Soc., Providence, R.I., 1978.

7. J. Franks, Homology and dynamical systems, CBMS Regional Conf. Ser. in Math., vol. 49, Amer. Math. Soc., Providence, R.I., 1982.

8. Nonsingular Smale flows on $S^{3}$, Topology 24 (1985).

9. C. Pugh and M. Shub, Embedding suspensions of subshifts of finite type in $S^{3}$, Contributions to Geometry and Analysis, C. Percelli and R. Sacksteder, eds., Johns Hopkins Univ. Press, Baltimore, Md., 1981.

10. D. Rolfsen, Knots and links, Publish or Perish, Berkeley, Calif., 1976.

11. S. Smale, On gradient dynamical systems, Ann. of Math. 74 (1961).

12. _ Differentiable dynamical systems, Bull. Amer. Math. Soc. 73 (1967).

13. R. Williams, Classification of subshifts of finite type, Ann. of Math. 98 (1973).

Department of Mathematics, Boston University, Boston, Massachusetts 02215 